\title{
Profils minces supercavitants à arrière tronqué
}

par J.-M. Michel

Chargé de Recherches au C.N.R.S.

Institut de Mécanique de l'Université Scientifique et Médicale de Grenoble et A. Rowe

Ingénieur de Recherches au C.N.R.S.

\section{III. - Etude physique du sillage en écoulement plan*}

\author{
par J.-M. MICHEL
}

Les modèles théoriques utilisés pour étudier les écoulements supercavitants reposent sur les principales hypothèses suivantes:

a) II existe, au voisinage du corps, une cavité où la pression est uniforme et constante et prend une valeur inférieure ou égale à sa valeur à l'infini aval. Cette valeur, considérée comme une donnée, ne dépend pas de la nature physique du fluide qui remplit la cavité.

b) Le fluide en mouvement est considéré comme parfait : on néglige la dissipation d'énergie due à la viscosité ou à la turbulence qui naît dans les régions où l'écoulement est instable (zone de fermeture de la cavité dans le schéma d'écoulement à jet rentrant de GILBARG) ; on néglige aussi les échanges d'énergie entre le fluide ambiant et celui de la cavité, échanges qui résultent principalement de la tension superficielle, de la viscosité et de la compressibilité.

Ces deux hypothèses complémentaires, outre les simplifications indispensables qu'elles apportent au traitement mathématique du problème, ont aussi pour conséquence d'éliminer du champ de la théorie l'analyse du mécanisme

* Les deux premières parties de cette étude ont paru dans la Houille Blanche, n० 3-1974, pages 205-214 et 215-230. par lequel, dans des conditions données, la pression dans la cavité s'établit à telle valeur. Cette question, qui ne se pose pratiquement pas dans le cas des cavités de vapeur, prend une importance considérable dans le cas des cavités ventilées : en cavitation de vapeur, les échanges de masse entre les deux phases sont très petits et $n^{\prime}$ influent que très peu sur la dynamique de l'écoulement ; c'est alors la température de l'eau qui, pour une vitesse et une pression de référence données, fixe la dépression relative $\sigma$ de la cavité. Au contraire, lorsque l'écoulement supercavitant est produit par l'injection d'air à l'arrière du profil, le paramètre $\sigma$ dépend de toutes les grandeurs d'entrée, particulièrement de la pression ambiante, de la forme du profil, de son incidence et du débit d'air injecté. La situation est alors analogue à celle que l'on rencontre dans le cas des sillages formés à l'aval d'obstacles non profilés, aux petits nombres de Reynolds : il apparaît, au voisinage du corps, une zone de recirculation dans laquelle la pression est presque uniforme; la valeur de la pression dépend essentiellement des échanges de masse et de quantité de mouvement à la frontière de cette zone, notamment dans sa partie aval. D'une manière semblable, dans le cas des cavités ventilées, dont on verra qu'elles présentent souvent un caractère globalement périodique, la valeur de la pression moyenne dans la cavité et la fréquence de pulsation sont reliées simul- 
tanément aux grandeurs d'entrée par la dynamique même de l'écoulement, dont les aspects les plus déterminants doivent être cherchés dans la zone de fermeture de la cavité.

Ces remarques sont importantes d'abord du point de vue des applications : la portance et la traînée d'un profil peuvent être exprimées en fonction du paramètre $\sigma$. Dans le cas des cavités ventilées et pour un profil très peu portant, ce paramètre est relié au débit d'air par une loi complexe où apparaissent des discontinuités et des boucles d'hystérésis. Dans le cas d'un profil portant, la relation est plus compliquée encore : la circulation induite autour du profil contribue à déterminer les conditions de fermeture de la cavité ; une variation très petite de l'incidence du profil peut modifier les conditions d'écoulement de l'air à l'aval de la cavité et changer notablement la valeur de $\sigma$.

Sur le plan théorique aussi la substitution du débit d'air à la pression moyenne de la cavité, comme grandeur déterminante de l'écoulement, a des conséquences importantes. Elle signifie en effet que l'on prend en compte la présence de l'air injecté dans les bases mêmes de l'analyse des aspects moyen et fluctuant de l'écoulement considérés en bloc. Dans ce cas la pression moyenne dans la cavité, ou le nombre $\sigma$, ne jouent que le rôle d'intermédiaires utiles pour le calcul : ce serait la tâche d'une théorie complète de les relier au débit d'air injecté. En d'autres termes, toute théorie qui considère l'écoulement fluctuant comme une petite perturbation d'un écoulement moyen et dans laquelle la masse d'air contenue dans la cavité fermée est constante, néglige un aspect essentiel du phénomène.

L'analyse physique globale des écoulements supercavitants joue alors un double rôle. En premier lieu, il s'agit de comparer les résultats expérimentaux à ceux des théories existantes en ce qui concerne la relation entre le paramètre $\sigma$ et certains paramètres globaux ; dans ce cadre, on peut laisser provisoirement en suspens le problème de la réalisation pratique de l'écoulement, par cavitation de vapeur ou par ventilation. Cette comparaison a été très largement effectuée dans le passé pour les coefficients d'efforts exercés par l'écoulement sur les profils. Nous présentons ici, en vue d'une telle confrontation, nos résultats concernant la longueur des cavités, grandeur qui caractérise globalement la géométrie des écoulements et qui ne semble pas avoir fait jusqu'ici l'objet d'une étude systématique.

En second lieu, afin de fournir les bases au modèle explicatif complet évoqué ci-dessus, l'analyse doit, dans le cas des cavités ventilées, préciser les paramètres qui déterminent l'écoulement et en déduire ses caractères physiques principaux. Nous présentons dans ce but nos résultats expérimentaux obtenus dans une configuration d'écoulement plan horizontal. Ils diffèrent sur quelques points des observations de SILBERMAN et SONG [1] à qui l'on doit la découverte et la description du phénomène de pulsation des cavités ventilées réalisé dans un jet libre vertical. La description cinématique de l'écoulement permet en particulier de mettre en évidence le rôle important joué conjointement par l'injection de l'air et par la condition de fermeture de la cavité. II semble qu'à cet égard la complexité du phénomène dépasse les mécanismes pris en compte dans les modèles théoriques de SONG [2], de HSU et CHEN [3] et de WOODS [4], ainsi que ceux que NAUDASCHER [5] a suggérés. A défaut d'une théorie complète tenant compte de l'écoulement des deux fluides, théorie qui semble encore difficile à élaborer même dans le cadre restreint du schéma du fluide parfait, l'analyse du mécanisme de pulsation suggère l'existence de deux chaînes de contre-réaction : on explique ainsi l'évolution interne de la cavité et son développement contre les forces d'inertie du sillage qui l'accompagne.

\section{1 - DISPOSITIF EXPÉRIMENTAL ET PARAME- TRES DE SIMILITUDE}

La majeure partie des essais a été effectuée dans le canal à surface libre du tunnel hydrodynamique de I'Université de Grenoble [6]. Les caractéristiques essentielles de ce canal sont les suivantes:

- hauteur d'eau : $280 \mathrm{~mm}$; largeur : $175 \mathrm{~mm}$; longueur $2500 \mathrm{~mm}$.

- pression absolue à la surface libre ajustable entre la tension de vapeur de l'eau et une pression de 2 bars;

- vitesse comprise entre 2 et $14,4 \mathrm{~m} / \mathrm{s}$;

- nombre de cavitation minimal voisin de 0,02 .

Le canal, dont les parois sont en verre, est enfermé dans un caisson dépressurisable équipé de grands hublots de visualisation. Le profil utilisé, de forme simple (coin symétrique de corde $60,5 \mathrm{~mm}$, de demi-angle $Y=80$ ) est placé sous la surface libre à une profondeur d'immersion de 7,14 ou $21 \mathrm{~cm}$.

Quelques essais ont été effectués dans une veine rectangulaire fermée (hauteur $300 \mathrm{~mm}$, largeur $125 \mathrm{~mm}$, longueur $1200 \mathrm{~mm})$, d'un tunnel appartenant à la SOGREAH * Dans cette veine, la vitesse pouvait être établie entre 1,5 et $8 \mathrm{~m} / \mathrm{s}$. Le profil était placé à mi-hauteur de la veine.

$\mathrm{p}_{\mathrm{o}}$

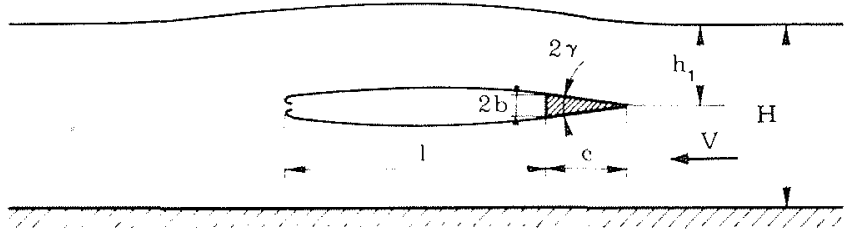

1/ Schéma de l'écoulement à surface libre

La figure 1 présente le schéma de l'écoulement autour d'un profil portant accompagné d'une cavité, placé dans le canal à surface libre.

Pour une telle configuration d'écoulement et pour un profil de forme donnée, les paramètres primaires de l'écoulement sont :

a) les paramètres géométriques :

- profondeur $\mathrm{H}$ du canal

- profondeur d'immersion $\mathrm{h}_{1}$

- corde c et incidence $\alpha$ du profil

* Devenue, depuis lors, la division "Technique des Fluides" des Etablissements ALSTHOM. 
b) la masse volumique $\rho$, seule grandeur qui caractérise le liquide. Pour l'analyse globale de l'écoulement que nous avons en vue, nous ne prenons pas en compte les forces dues aux contraintes visqueuses et à la tension superficielle ; celles-ci devraient être introduites dans l'analyse détaillée du sillage de la cavité et des fluctuations des surfaces libres. De plus, nous supposons que la température de l'eau est voisine de la température ambiante et nous négligeons ses variations; la tension de vapeur $p_{v}$ de l'eau est donc supposée constante.

c) les paramètres dynamiques :

- intensité g de la pesanteur

- pression $p_{0}$ à la surface libre

- vitesse $V$ d'entrée du fluide

- débit-masse d'air $Q$ dans le cas des cavités ventilées.

Dans le régime d'écoulement étudié, le profil est accompagné d'une cavité de longueur $\ell$ où la pression s'établit à une valeur $\mathrm{p}_{\mathrm{c}}$ :

- en cavité de vapeur, $p_{c} \cong p_{v}{ }^{*}$

- en cavité ventilée, $p_{c}=p_{v}+P_{\text {air }}$

Dans ce dernier cas, la longueur de la cavité oscille entre une valeur minimale $\ell_{m}$ et une valeur maximale $\ell_{M}$ et la pression varie d'une manière quasi sinusoïdale. On considère alors les valeurs moyennes $\bar{l}$ et $\bar{p}_{c}=p_{v}+\bar{p}_{\text {air }}$ et l'on forme la dépression de la cavité $\Delta \bar{p}=\left(p_{0}+\rho g_{1}\right)$ $-\bar{p}_{\mathrm{c}}$.

L'écoulement moyen est caractérisé par les grandeurs secondaires $\bar{l}$ et $\Delta \bar{p}$ que l'on exprime en fonction des grandeurs primaires. On doit cependant, dans le cas des cavités ventilées, mettre en évidence la pression moyenne de l'air dans la cavité comme paramètre secondaire privilégié : sous forme adimensionnelle, et après l'élimination des paramètres les moins significatifs, tels que le nombre de Froude (les vitesses étant supposées grandes) ou la profondeur relative $\mathrm{H} / \mathrm{c}$ de l'écoulement (gardée constante dans nos essais), on obtient :

$$
\sigma_{a}=\frac{\bar{P}_{\text {air }}}{\rho \frac{v^{2}}{2}}=F_{1}\left[\frac{h_{1}}{c}, \alpha, \sigma_{v}, C_{Q}\right]
$$

avec $\sigma_{v}=\frac{p_{0}+\rho g h_{1}-p_{v}}{\rho \frac{v^{2}}{2}}$

et

$$
\mathrm{C}_{\mathrm{Q}} \text { : coefficient de débit d'air }
$$

(b) $\sigma=\frac{\Delta \bar{p}}{p \frac{v^{2}}{2}}=\sigma_{v}-\sigma_{a}=F_{2}\left[\frac{h_{1}}{c}, \alpha, \sigma_{v}, c_{Q}\right]$

\footnotetext{
* La pression dans la cavité diffère par excès de la tension de vapeur d'une quantité très faible. Contrairement aux résultats de BRENNEN [2], nous n'avons pu établir une corrélation entre cette différence et la teneur en air de l'eau.
}

(c) $\quad \frac{\bar{l}}{c}=F_{3}\left[\frac{h_{1}}{c}, \alpha, \sigma_{v}, c_{Q}\right]$

Si l'on élimine $\mathrm{C}_{\mathrm{Q}}$ entre les relations (b) et $(\mathrm{c})$ on obtient la relation :

$$
\frac{\bar{\ell}}{c}=F_{4}\left[\frac{h_{1}}{c}, \alpha, \sigma\right]
$$

où l'on a fait disparaître aussi le paramètre $\sigma_{v}$ dont l'expérience et l'analyse directe montrent qu'il n'a pas d'influence sur $\bar{l}$. La relation (d) est utile pour comparer les résultats théoriques aux résultats expérimentaux, aussi bien en écoulement ventilé qu'en cavitation naturelle. Elle offre aussi l'avantage d'éliminer les discontinuités que l'on rencontre dans les lois $\sigma\left(C_{Q}\right)$ et $\bar{l}\left(C_{Q}\right)$. Enfin, elle permet de caractériser l'écoulement moyen soit par $\sigma$ soit par $\ell / c$, grandeur assez aisément évaluable. Les relations (b) et (c) font apparaître la dépendance de $\sigma$ et $\bar{l} / c$ non seulement à l'égard du débit d'air, mais aussi à l'égard de l'incidence et des conditions générales de l'écoulement par rapport à la cavitation naturelle, caractérisée par le paramètre $\sigma_{v}$.

Dans le cas des cavités ventilées, l'écoulement fluctuant est caractérisé principalement par la fréquence $f$ du phénomène. L'expérience conduit à former le nombre adimen-

sionnel $\varphi=f / \sqrt{\frac{\bar{P}_{\text {air }}}{\rho c \bar{l}}}$ et à l'exprimer, par des substitutions analogues à celles qui précèdent, sous la forme :

$$
\varphi=F_{5}\left[\frac{h_{1}}{c}, \alpha, \frac{\bar{l}}{c}, \frac{\sigma}{\sigma_{a}}\right]
$$

avec

$$
\frac{\sigma}{\sigma_{a}}=\frac{\Delta \bar{p}}{\bar{P}_{\text {air }}}
$$

Quelques remarques peuvent être faites à propos de la relation (e) qui permet seule de regrouper convenablement les points expérimentaux. Le paramètre $\sigma / \sigma_{\mathrm{a}}$ y représente le rapport de la différence $\Delta \bar{p}$ des pressions, responsables de l'écoulement moyen, à la pression de l'air dans la cavité, auquel on peut attribuer le caractère fluctuant de l'écoulement. La présence de la longueur moyenne $\bar{\ell}$ dans l'expression de $\varphi$ montre l'importance de ce paramètre intermédiaire dans l'analyse des écoulements à cavités ventilées. Enfin, la mise en évidence du terme $\bar{P}_{\text {air }}$ laisse ouverte la possibilité de deux mécanismes : pour un gaz donné évoluant de manière barotrope, la compressibilité du gaz et sa masse volumique, qui caractérise son inertie, $s^{\prime}$ expriment toutes deux en fonctiondu seul terme pression*.

\footnotetext{
* Dans la suite, les essa is ayant été effectués à incidence moyenne nulle, nous ne ferons pas apparaitre l'influence de $\alpha$ sur les diverses grandeurs analysées.
} 


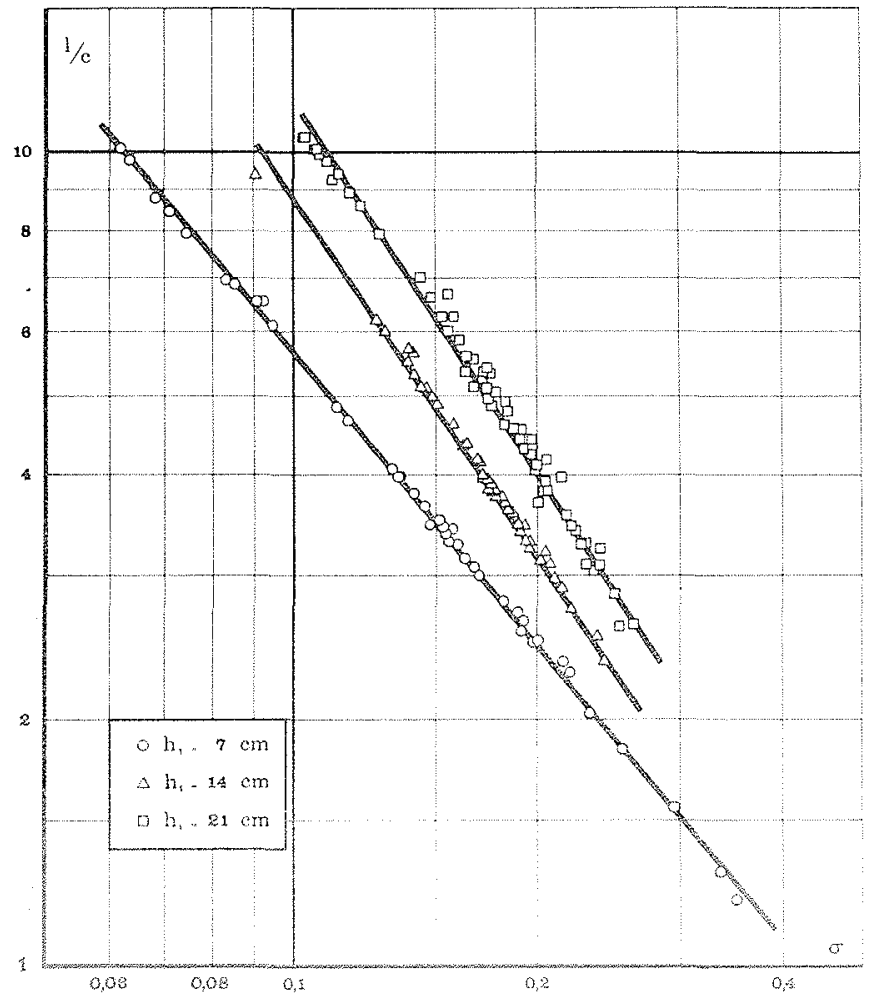

2 f cavises de rapew :

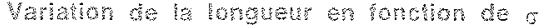

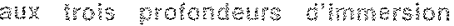

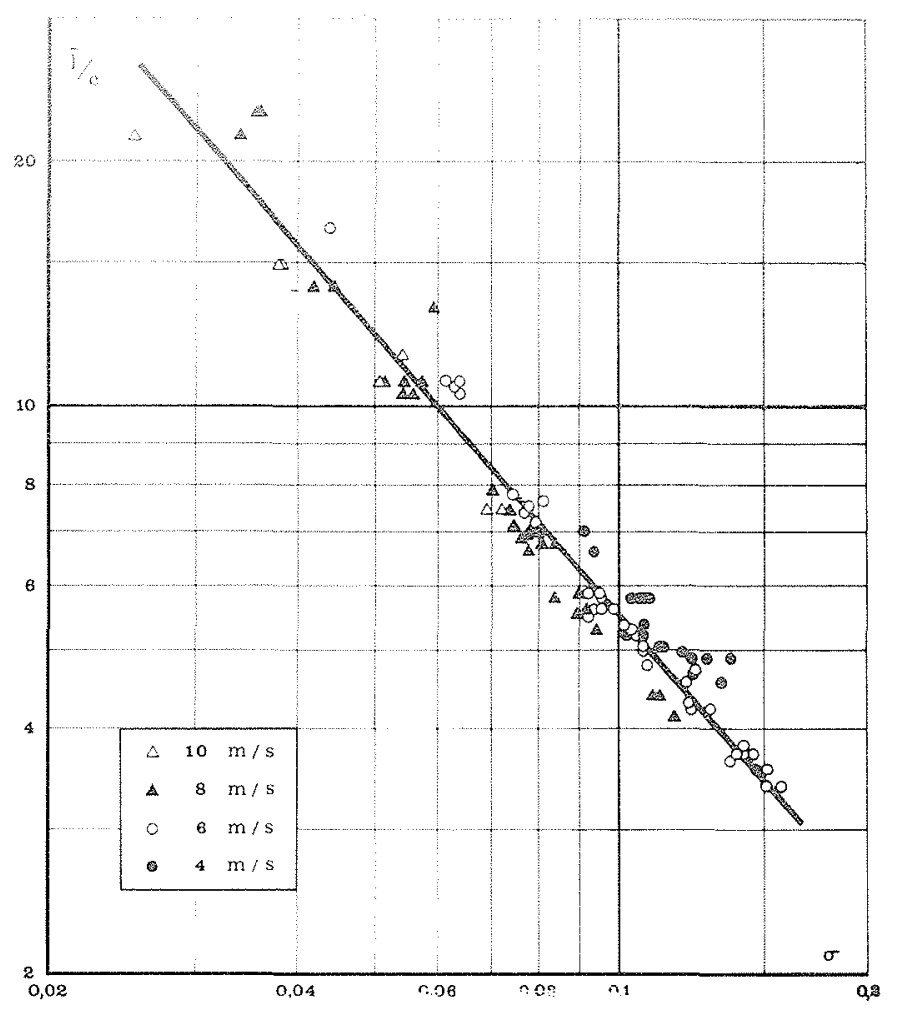

$3 /$ Cavités ventilées:

longueur moyenne en fonction de Profondeur $h_{1}=7 \mathrm{~cm}$

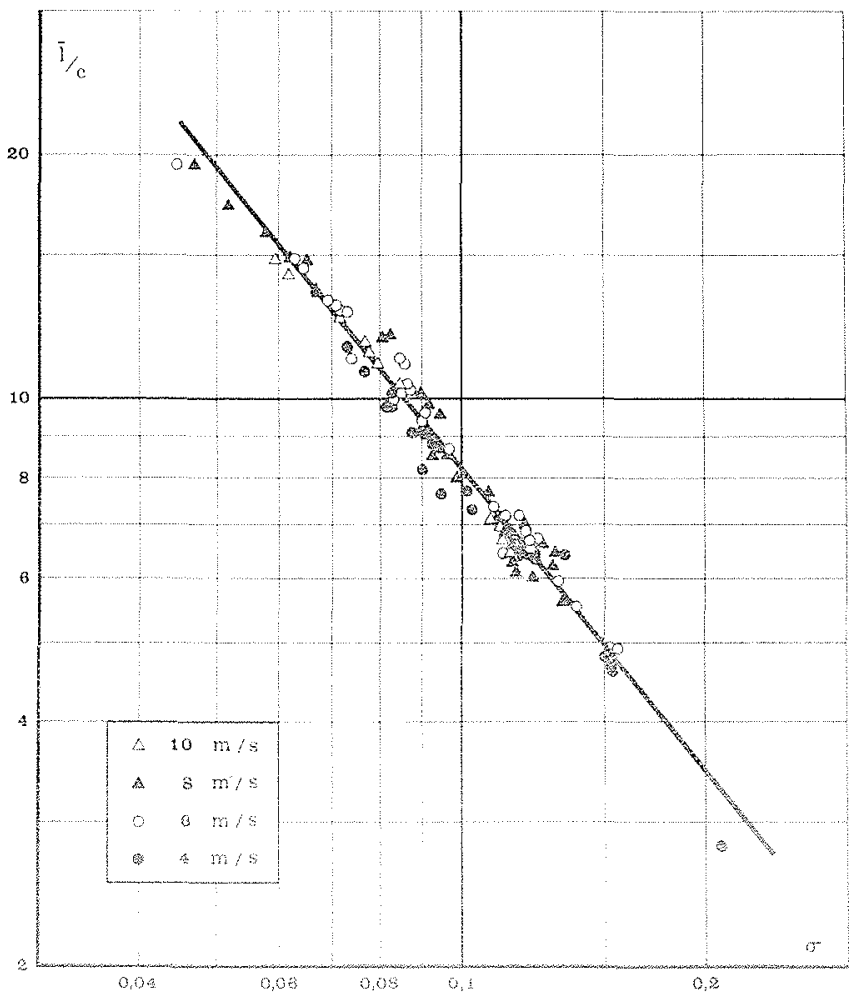

a) raveres ventos :

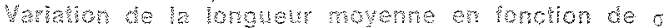

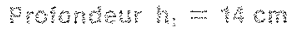

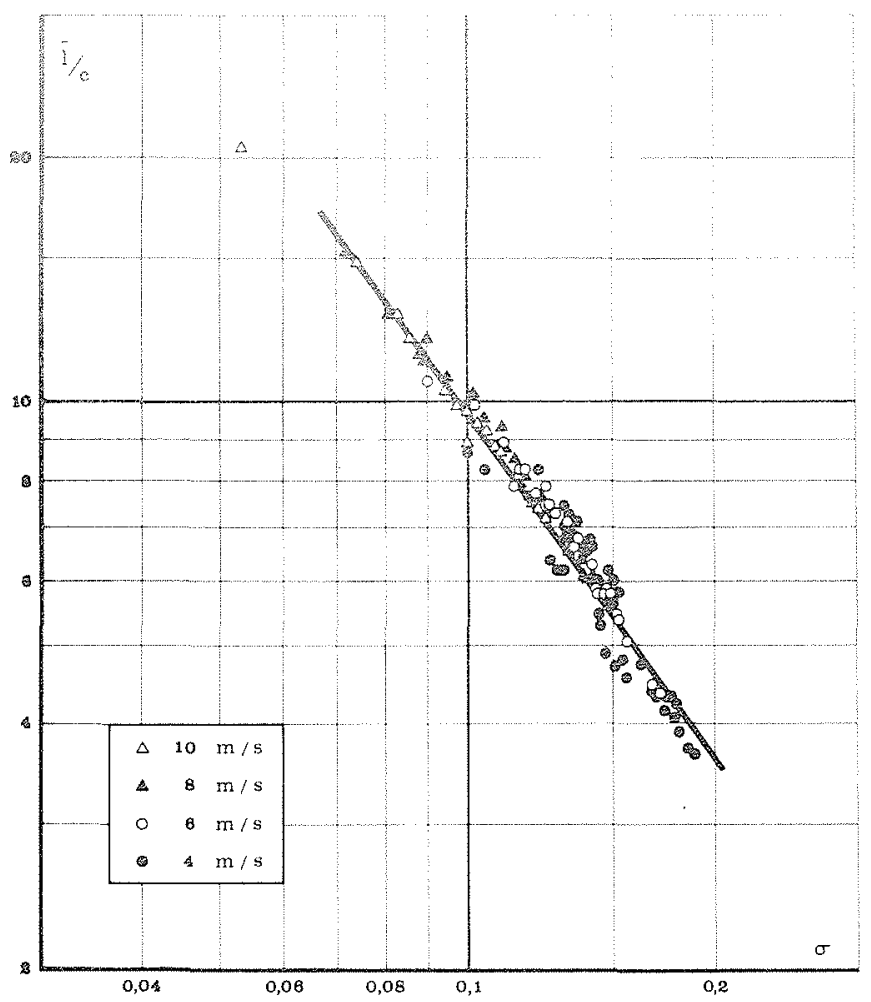

$5 /$ Cavités ventilées:

Varlation de la longueur moyenne en fonction de Profondeur $h_{1}=21 \mathrm{~cm}$ 


\section{2 - RÉSULTATS}

\subsection{Longueur des cavités}

L'expérience montre que, dans le domaine des vitesses utilisées pour les essais, on obtient un regroupement convenable des points lorsque, pour une profondeur relative $h_{1} / c$ donnée, on représente la longueur relative $l / c$ (cavités de vapeur) ou $\bar{l} / \mathrm{c}$ (cavités ventilées), en fonction du nombre de cavitation $\sigma$. Ce fait montre que la gravité n'a pratiquement pas d'influence sur la longueur des cavités.

L'analyse théorique montre que dans le cas d'un écoulement illimité la longueur de la cavité suit une loi en $\sigma^{-2}$ lorsque le nombre $\sigma$ est petit. Par analogie, nous particularisons la loi (d) sous la forme :

$$
\left.\frac{l}{c} \text { (ou } \frac{\bar{l}}{\mathrm{c}}\right) \simeq A \quad \sigma^{-n}
$$

dans laquelle les constantes $A$ et $\mathbf{n}$ doivent dépendre de la profondeur relative $h_{1} / c$. Cette hypothèse est assez bien satisfaite dans la gamme des mesures effectuées puisqu'en coordonnées logarithmiques les points de mesure sont à peu près alignés comme le montrent les figures 2 (cas des cavités de vapeur) et les figures 3 à 5 (cas des cavités ven. tilées). La dispersion plus grande des points dans le cas des cavités ventilées est due surtout à la mauvaise définition de la longueur maximale \&M. Les points de la figure 2 ont été obtenus pour des vitesses comprises entre 4 et $14 \mathrm{~m} / \mathrm{s}$

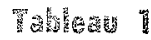

Cavités de vapeur: Valeurs de $A$ et $n$

\begin{tabular}{|c|c|c|c|c|c|}
\hline $\begin{array}{l}n \\
n n\end{array}$ & A & $n$ & N & $r$ & $\begin{array}{l}\text { Domaine do } \\
\text { waritutions de } \frac{1}{8}\end{array}$ \\
\hline 7 & 0.38 & 1,20 & 41 & $=0,990$ & $1.2<\frac{1}{6}<10.1$ \\
\hline 14 & 0.31 & 1,46 & 45 & $=0.987$ & $2,3<\frac{1}{6}<9,4$ \\
\hline 21 & 0.36 & 1,51 & 62 & $=0.894$ & $2,8<\frac{1}{6}<10,1$ \\
\hline
\end{tabular}

\section{Tableag 2}

Cavités ventllés: Valours do $A$ et $n$

\begin{tabular}{|c|c|c|c|c|c|}
\hline $\begin{array}{c}h_{1} \\
n\end{array}$ & $A$ & $n$ & $N$ & $r$ & $\begin{array}{c}\text { Domnino de } \\
\text { varintions de }\end{array}$ \\
\hline 7 & 0.38 & 1,16 & 99 & $-0,877$ & $3,4<\frac{1}{c}<23$ \\
\hline 14 & 0,45 & 1,26 & 109 & $-0,985$ & $2,8<\frac{1}{c}<19,4$ \\
\hline 21 & 0,34 & 1,47 & 142 & $-0,980$ & $3,6<\frac{j}{0}<20,6$ \\
\hline
\end{tabular}

On peut évaluer les constantes $A$ et $n$ de la loi (1) à partir des équations des droites des moindres carrés correspondant à l'ensemble des points de mesure. Les tableaux 1 et 2 résument les résultats pour les trois profondeurs d'immersion. On $y$ a fait figurer aussi le nombre $N$ de points de mesure, la valeur du coefficient de corrélation $r$ qui caractérise la linéarité de la relation dans le diagramme logarithmi. que et le domaine de variation de $\ell / c$ ou $\bar{l} / \mathrm{c}$.

Les figures 2 à 5 et les deux tableaux ci-dessus mettent en évidence l'influence de la profondeur d'immersion sur la longueur des cavités. Elles montrent aussi qu'à nombre de cavitation égal les cavités de vapeur sont un peu plus longues que les cavités ventilées, au moins dans le domaine des valeurs de oétudiées. La traduction quantitative de ces faits, que l'on tente par l'évaluation de $A$ et $n$, ne fait apparaitre clairement que la croissance de $n$ en fonction de $h_{1}$. II semble que lorsque $h_{1} / c$ devient petit, l'exposant $n$ tende vers l'unité. On peut justifier ce fait [8] par un modèle très simple dans lequel le coin, placé à l'incidence d'adaptation $-\alpha_{2}$, est suivi d'une cavité dont la longueur est déterminée essentiellement par le comportement de la nappe superieure. Si on assimile celle-ci à un jet soumis aux pressions po et po et prenant de ce fait une forme circulaire, on trowve une loi du type: $\ell / c \simeq 4 \frac{h_{1}}{c} \frac{\alpha+\alpha a}{\sigma}$.

FOUILLET $[g \mid$ a rerrouvé, au terme d'une thérie asymp. tomatique des écoulements supercavitants, la loi en $\sigma^{-1}$ sous une forme legerement differente:

$$
2 / c \simeq \frac{2}{6} \sqrt{\frac{n_{1}}{c}} \bar{T}
$$

ou $\bar{T}$ designe le coefficient de tranée.

\section{2- Snlage des cantres de wapen}

La photographie de la figure 6 donne une image instantané de la partie arritre d'une cavité de vapeur et de son slliage proche. On y ramarque particulièrement l'aspect tourbillonnaire du sillage rendu visible par des filaments de vapeur non hractionnés en petites bulles. On pourrait en déduire qu'il existe une fréquence caractéristique ; cependant, il $n^{\prime}$ á pas été possible de détecter nettement un phénomène périodique ; par un moyen optique, on a pu seulement mettre en évidence des suites de quelques bouffées turbulentes (moins d'une dizaine), se suivant à intervalles presque réguliers, de l'ordre de 11 millisecondes, pour une vitesse $\mathrm{V}=8 \mathrm{~m} / \mathrm{s}, \sigma$ étant égal à 0,190 . II $\mathrm{y}$ aurait donc une émission discontinue de trains d'ondes indépendants, interrompue peut-être par le fait du jet rentrant aléatoire.

La turbulence du sillage, due principalement à l'instabilité de l'écoulement dans la partie arrière de la cavité, a pour effet de réagir sur le corps maintenu à pression constante que constitue celle-ci et de lui imposer, dans sa partie arrière, des oscillations transversales très rapides et de très petite amplitude. Elle a aussi pour conséquence, conjointement avec l'émission de bulles de vapeur à l'arrière de la cavité, de diminuer la surpression dans la zone de fermeture. En l'absence de turbulence et pour un écoulement permanent répondant par exemple au schéma de GILBARG, il existe un point d'arrêt où la pression PA est en excès sur la pression de référence de la pression dynamique $\rho \vee 2 / 2$. 
Expérimentalement, on peut définir un défaut adimensionnel de pression $P$ par la relation

$$
\frac{\mathrm{pA}-\mathrm{pr}}{\frac{1}{2} \rho \mathrm{V}^{2}}=1-\mathrm{P}, \quad \mathrm{P}<1
$$

Le coefficient $P$ peut être considéré comme un indice de la validité du schéma théorique dans la partie aval de la cavité. La valeur de $P$, mesurée lors de deux essais menés dans des conditions différentes de pression et de vitesse, pour $h_{1}=14 \mathrm{~cm}, \sigma=0,170, l \simeq 23 \mathrm{~cm}$, a été trouvée de l'ordre de 0,88 . La surpression à l'arrière de la cavité n'est donc dans ces conditions que de $12 \%$ de sa valeur théorique.

\section{3 - Cavités ventilées. Aspect périodique}

\subsection{1 - Description du phénomène}

Le phénomène de pulsation des cavités ventilées a été largement décrit pas SILBERMAN et SONG. Nous ne reprenons cette description que dans la mesure où elle est nécessaire à la suite de cet exposé et où quelques différences dans le comportement des écoulements, dues à la différence de leur configuration d'ensemble, ont pu être constatées.

Lorsqu'on injecte de l'air à la base du profil, l'écoulement présente divers aspects qui sont, particulièrement pour leur caractère périodique, sous la dépendance des paramètres $\sigma_{v}$ et $\sigma / \sigma_{a}$.

Lorsque la valeur $p_{0}$ de la pression à la surface libre du canal est très petite, de sorte que $\sigma_{v}$ est très inférieur à un seuil $\sigma_{\text {vo }}$ dont la valeur est d'environ 0,30 à 0,45 , une très faible injection d'air donne une longue cavité sans aucun caractère périodique : la cavité passe d'une longueur à une autre en demeurant relativement stable pendant un temps de quelques secondes : l'air s'échappe d'une manière aléatoire à l'arrière de la cavité.

Lorsque $\sigma_{V}$ est soit à peu près égal, soit supérieur à $\sigma_{\text {vo }}$, une très faible injection d'air ne modifie d'abord que très peu l'aspect de l'écoulement : s'il existe initialement une petite cavité formée d'une émulsion d'eau et de vapeur, l'air s'échappe à l'arrière sous forme de petites bulles comme en cavitation de vapeur ; en l'absence de cavité de vapeur initiale, l'air injecté se mélange à l'eau dans le sillage du du profil; il est alors évacué sous forme de petites bulles qui se localisent au cœur des tourbillons alternés issus des bords de fuite du profil. Notons ici que, à la différence de SILBERMAN et SONG, nous n'avons pu augmenter la longueur de la cavité dans ce régime précédant le régime pulsant.

Une légère augmentation du débit d'air a pour effet d'augmenter brutalement la longueur de la cavité et de faire passer au régime de cavité pulsante. Ce changement de régime se produit lorsque le nombre $\sigma / \sigma_{a}$ devient inférieur à une valeur voisine de 0,31. Dans les expériences de SILBERMAN et SONG, la valeur correspondante de $\sigma / \sigma_{v}$, proche de 0,20 , est un peu inférieure à la nôtre, égale à 0,23. L'augmentation de la longueur de la cavité, qui semble manifester une instabilité globale de l'écoulement, est plus grande lorsque $\sigma_{v}$ est plus petit. Pour $\sigma_{v} \simeq \sigma_{\text {vo }}$, on peut obtenir $\bar{l} / \mathrm{c} \simeq 10$ ou 15 . Dans ce cas, on peut observer des oscillations verticales de la surface libre de l'écoulement.

Nous décrivons maintenant le phénomène de pulsation en supposant le nombre $\sigma_{V}$ supérieur à $\sigma_{v o}$ : la majeure partie de nos essais a été effectuée dans ces conditions.

La cavité, dont la longueur évolue entre la valeur minimale $\ell_{m}$ et la valeur maximale $\ell_{M}$, présente alors divers aspects, caractérisés par le nombre $k$ de longueurs d'ondes, d'où les régimes $R_{k}$ (fig. 7) (le terme "longueur d'onde" est utilisé seulement par commodité ; il ne signifie pas que les perturbations des frontières de la cavité résultent d'un échange d'énergie cinétique et d'énergie potentielle du liquide). Le passage d'un régime à un autre s'effectue $d^{\prime}$ 'une manière brutale pour certaines valeurs-seuil du débit d'air $Q$, différentes lorsqu'on augmente le débit ou lorsqu'on le diminue (fig. 8), d'où le caractère hystérétique du diagramme $\sigma(Q)$. L'approche de la limite d'un régime se manifeste par des fluctuations importantes de la pression moyenne dans la cavité : la limite de régime traduit une instabilité de l'écoulement. Le passage d'un régime à un autre se manifeste par une petite diminution de la fréquence, alors que la longueur d'onde $\lambda$, qui sera définie plus loin, reste sensiblement constante.

Lorsque le débit d'air est augmenté à l'extrême, les phénomènes périodiques disparaissent: l'écoulement de l'air se fait soit par des grosses bulles qui se détachent de la frontière supérieure de la cavité pour des vitesses pas trop grandes (inférieures à $6 \mathrm{~m} / \mathrm{s}$ environ) soit, pour les vitesses plus élevées, par des bulles qui s'échappent surtout à l'aval de la cavité.

Deux mécanismes contribuent au détachement des paquets d'air à l'aval des cavités dans le régime pulsant : d'une part le développement, vers l'intérieur de la cavité, des perturbations de ses frontières qui tendent à se rejoindre à l'aval ; d'autre part, le jet rentrant, manifesté principalement par l'émulsionnement, à partir de l'aval, de la partie arrière de la cavité. Aux vitesses les plus petites, le premier mécanisme semble prépondérant, de sorte que les instants où la cavité atteint sa longueur minimale et sa longueur maximale sont confondus; aux plus grandes vitesses, l'émulsionnement envahit rapidement la partie arrière de la cavité jusqu'au point où sont parvenues, sans nécessairement se rejoindre, les perturbations des frontières de la cavité. Dans ce cas les deux instants sont décalés d'une valeur très petite par rapport à la période $T$ de pulsation. La vitesse de l'air dans la cavité est toujours inférieure à la vitesse de l'eau. Elle est susceptible de varier beaucoup lorsqu'un régime est établi et que le nombre $\sigma_{V}$ est fixé. Par exemple, pour $V=8 \mathrm{~m} / \mathrm{s}, \sigma_{v} \simeq 0,95, h_{1}=14 \mathrm{~cm}$, en régime à une longueur d'onde, elle varie entre 0,41 et $2,40 \mathrm{~m} / \mathrm{s}$ lorsque le débit d'air est augmenté. Le passage à un régime supérieur se traduit par une diminution de sa valeur.

Dans les conditions d'essais utilisées, le domaine de variation de la fréquence de pulsation s'étend entre 8 et 45 $\mathrm{Hz}$ environ. Pour $\mathrm{V}$ et $\mathrm{h}_{1}$ fixés et pour plusieurs valeurs du nombre $\sigma_{V}$, on obtient un diagramme d'évolution de la fréquence en fonction de $\sigma$ tel que celui de la figure 9 . Les points expérimentaux correspondant à tous les régimes sont dispersés dans le plan $(\sigma, f)$. Pour la clarté de la figure, on 
6/ Vue d'une cavité de vapeur

et de son sillage

$\mathrm{h}_{1}=14 \mathrm{~cm}$

$v=12 \mathrm{~m} / \mathrm{s}$

$\sigma=0,139$

7/ Forme de la cavité à l'instant

ou elle atteint

sa longueur minimale

8/ Evolution-type

du débit-masse d'air

en fonction de $\sigma$

(V et $\sigma r$ constants)

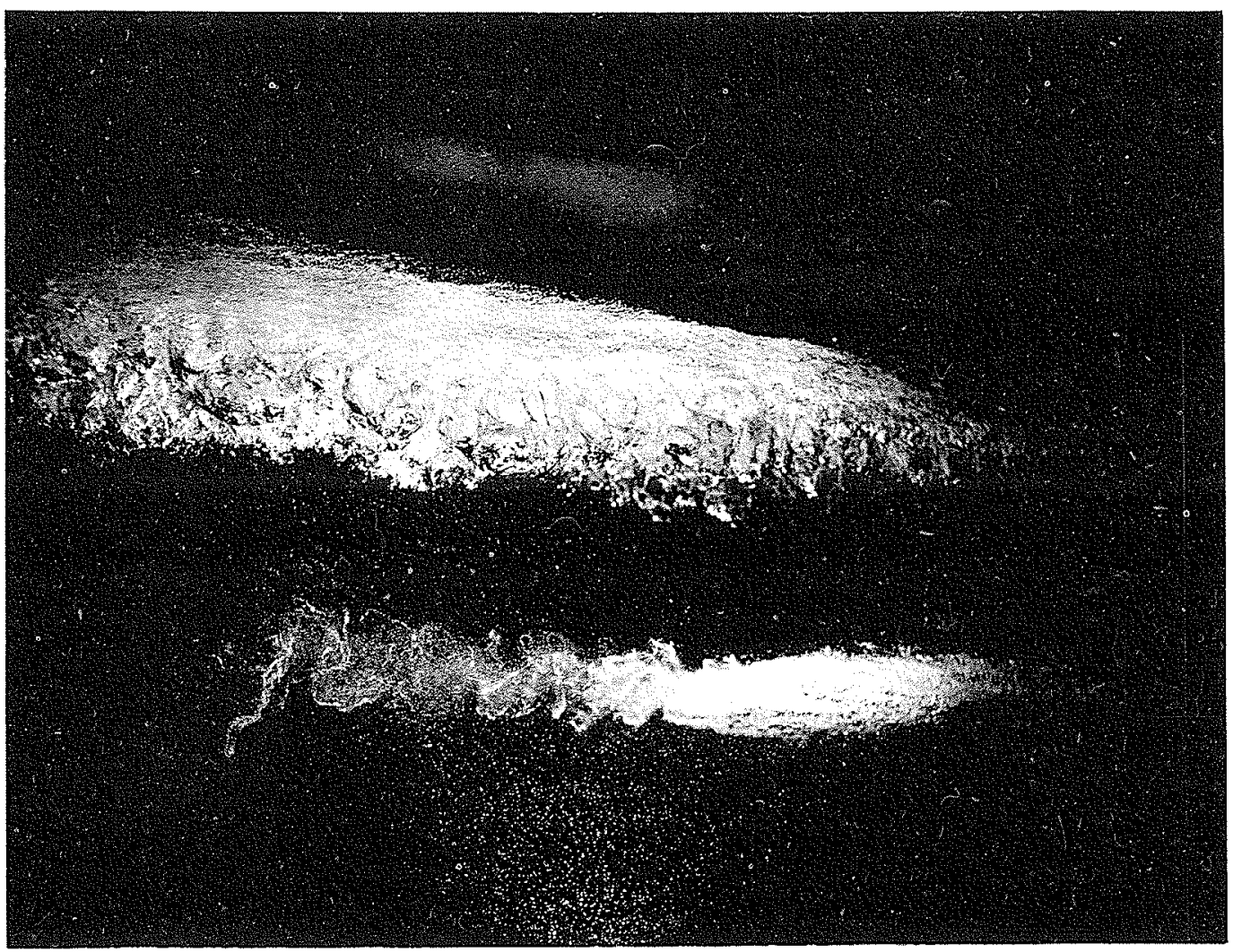

\section{6/}

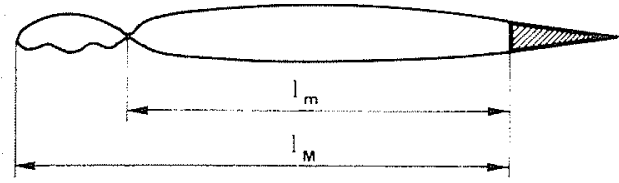

a - Règime à une longueur d'onde $\left(R_{1}\right)$

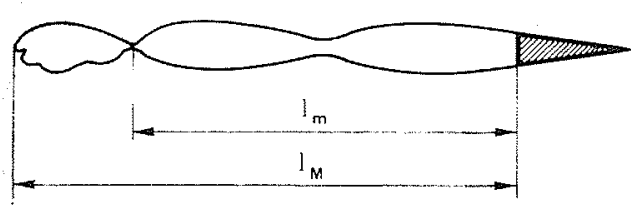

b- Régime à deux longueurs d'ondes $\left(R_{2}\right)$

\section{$7 /$}
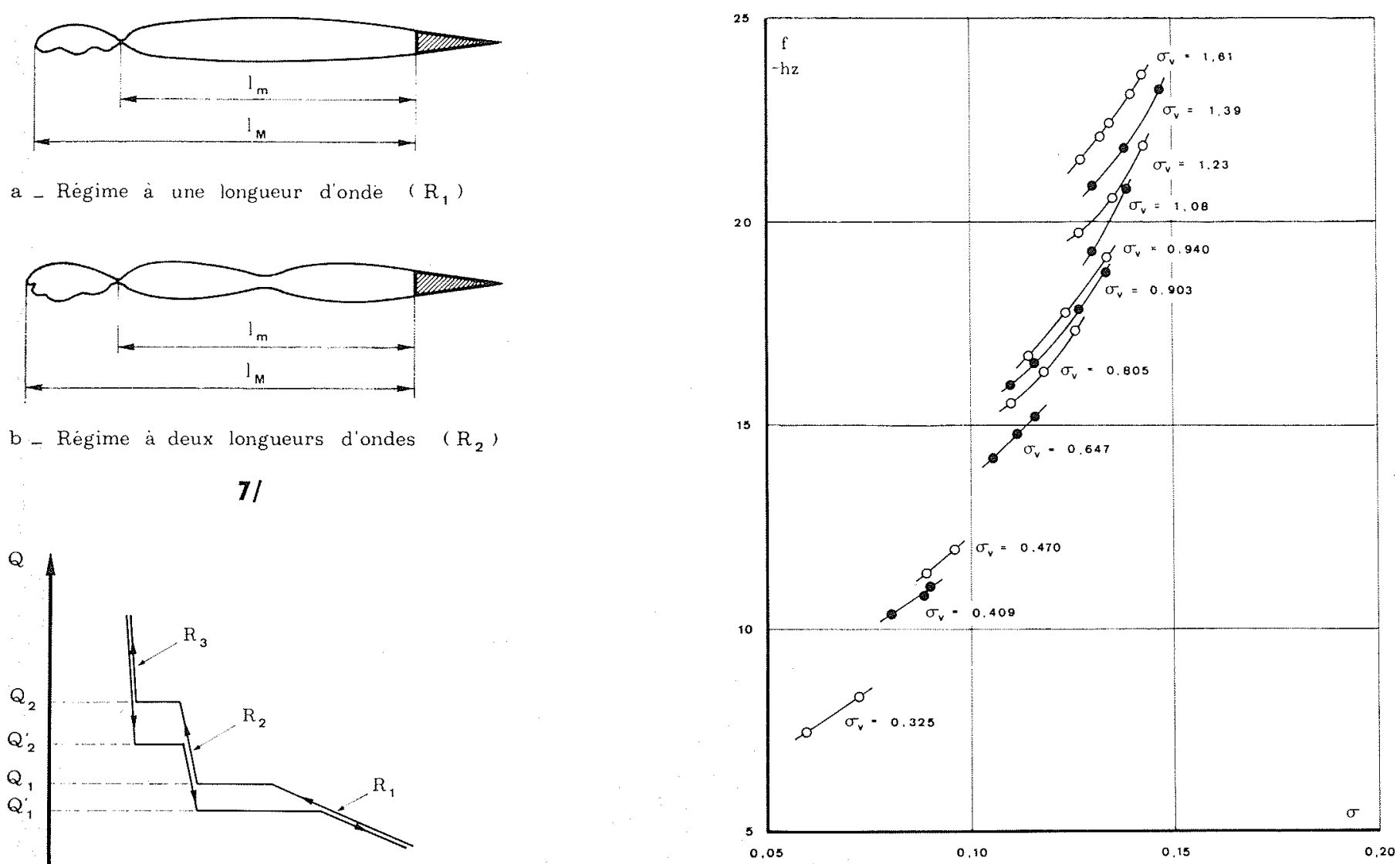

9/ Variation de la fréquence en fonction de pour des valeurs croissantes de $\sigma$ Régime $R_{1}-h_{1}=21 \mathrm{~cm}-V=8 \mathrm{~m} / \mathrm{s}$ 


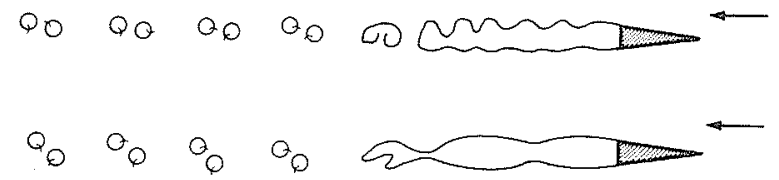

10/ Influence de la gravité sur la direction du jet rentrant ef sur la disposition des tourbillons dans le sillage

s'est limité à la représentation des points correspondants au régime à une longueur d'onde. Lorsqu'on maintient constante la vitesse $V$ ainsi que le paramètre $\sigma$ lou la longueur moyenne $\bar{l}$ ), la fréquence varie approximativement comme $\sqrt{\sigma_{a}}$; ce fait a fondé l'analyse donné dans le paragraphe
(relation e).

La gravité se manifeste de plusieurs manières : elle contribue à amplifier les ondes sur la paroi supérieure de la cavité ; elle rend la ligne moyenne de la cavité légèrement ascendante vers I'aval; enfin elle tend à dévier le jet rentrant vers le haut. Les schémas de la figure 10 illustrent ce dernier effet que l'on doit associer à la portance produite par l'effet de la gravité ; en a, le jet est presque vertical (cas des très faibles vitesses, inférieures à $2 \mathrm{~m} / \mathrm{s}$ ) ; en $b$, le jet est oblique (cas des vitesses comprises entre 2 et $5 \mathrm{~m} / \mathrm{s}$ environ). II en résulte dans le sillage des paquets d'air de forme caractéristique, que l'on peut schématiser par des tourbillons apairés dont les centres sont soit sur la ligne moyenne

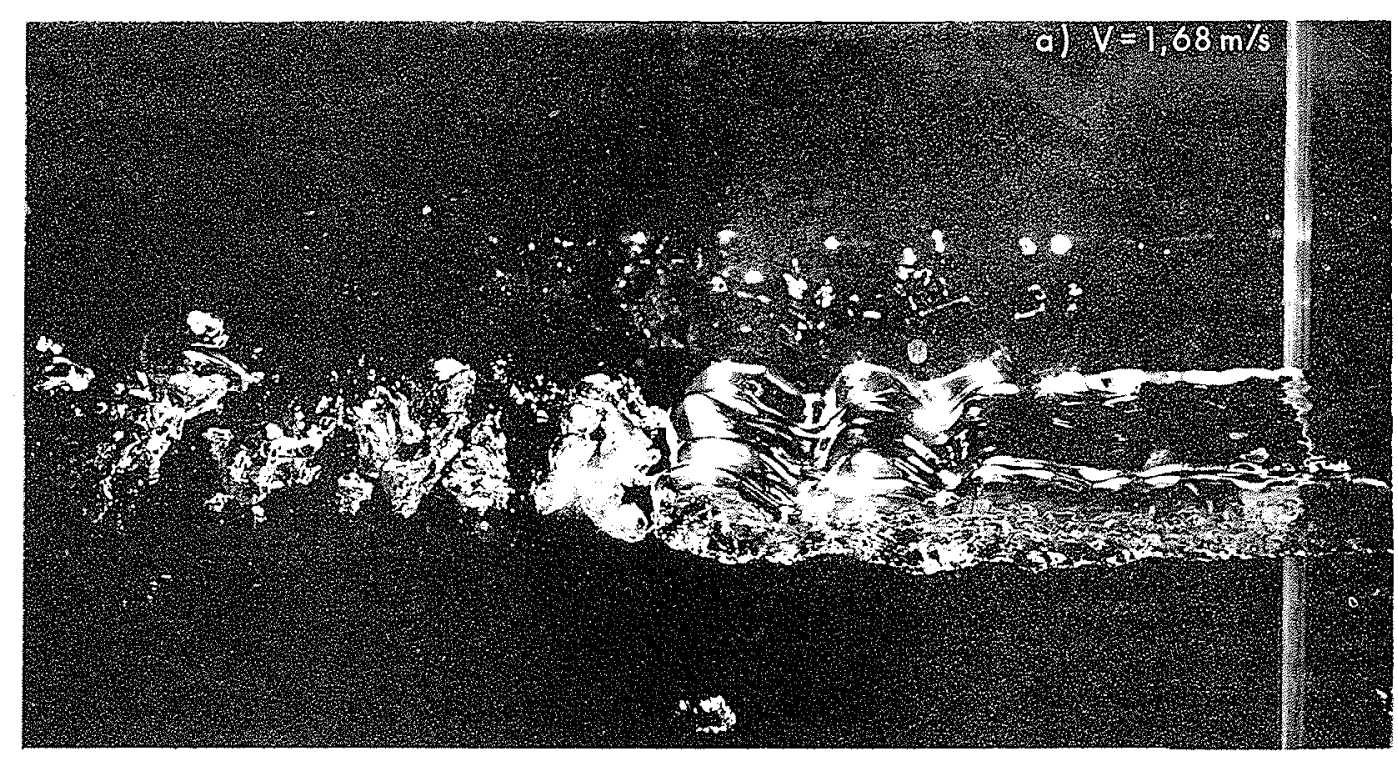

II/ Vue de l'écoulement à petit nombre de Froude

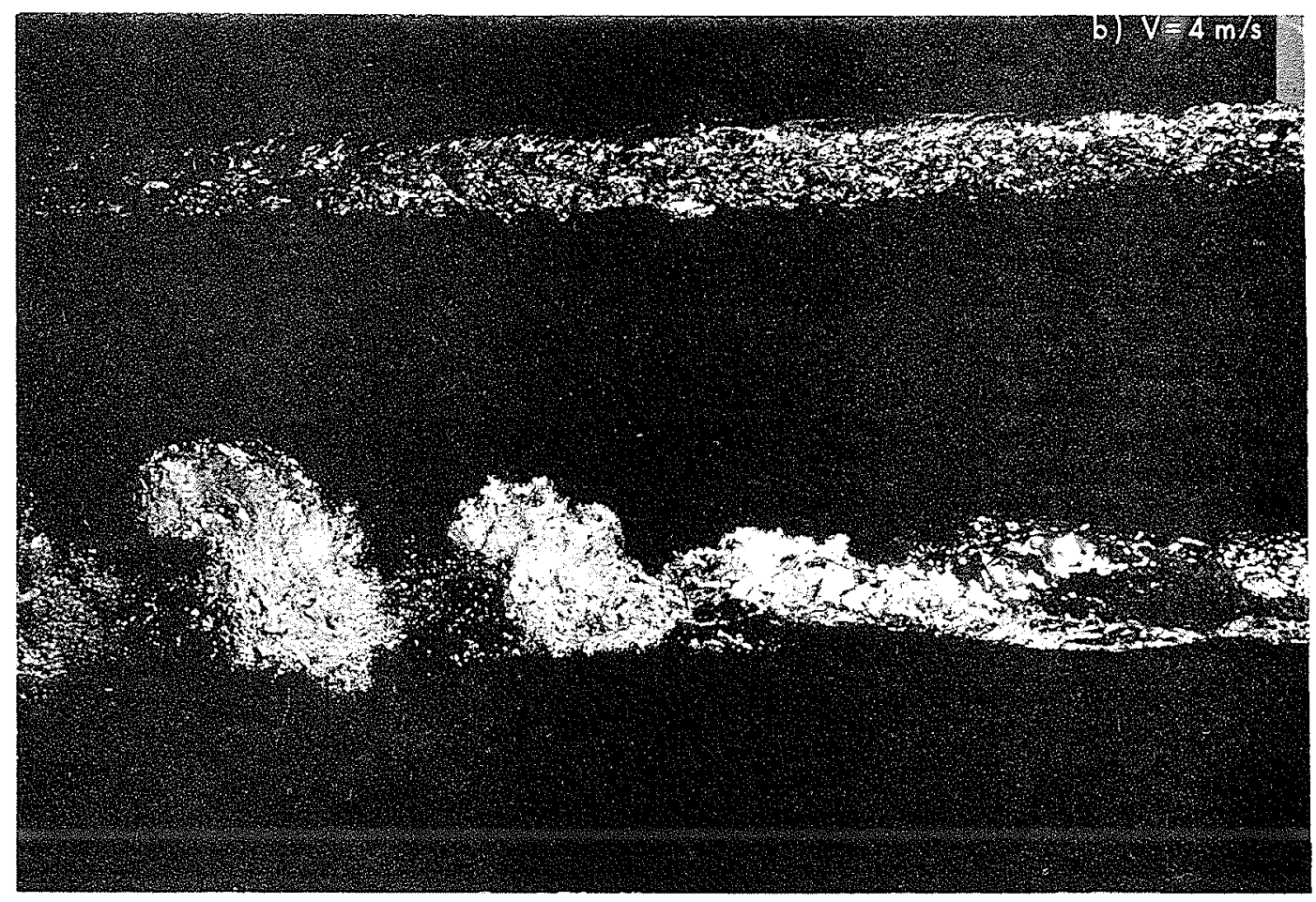




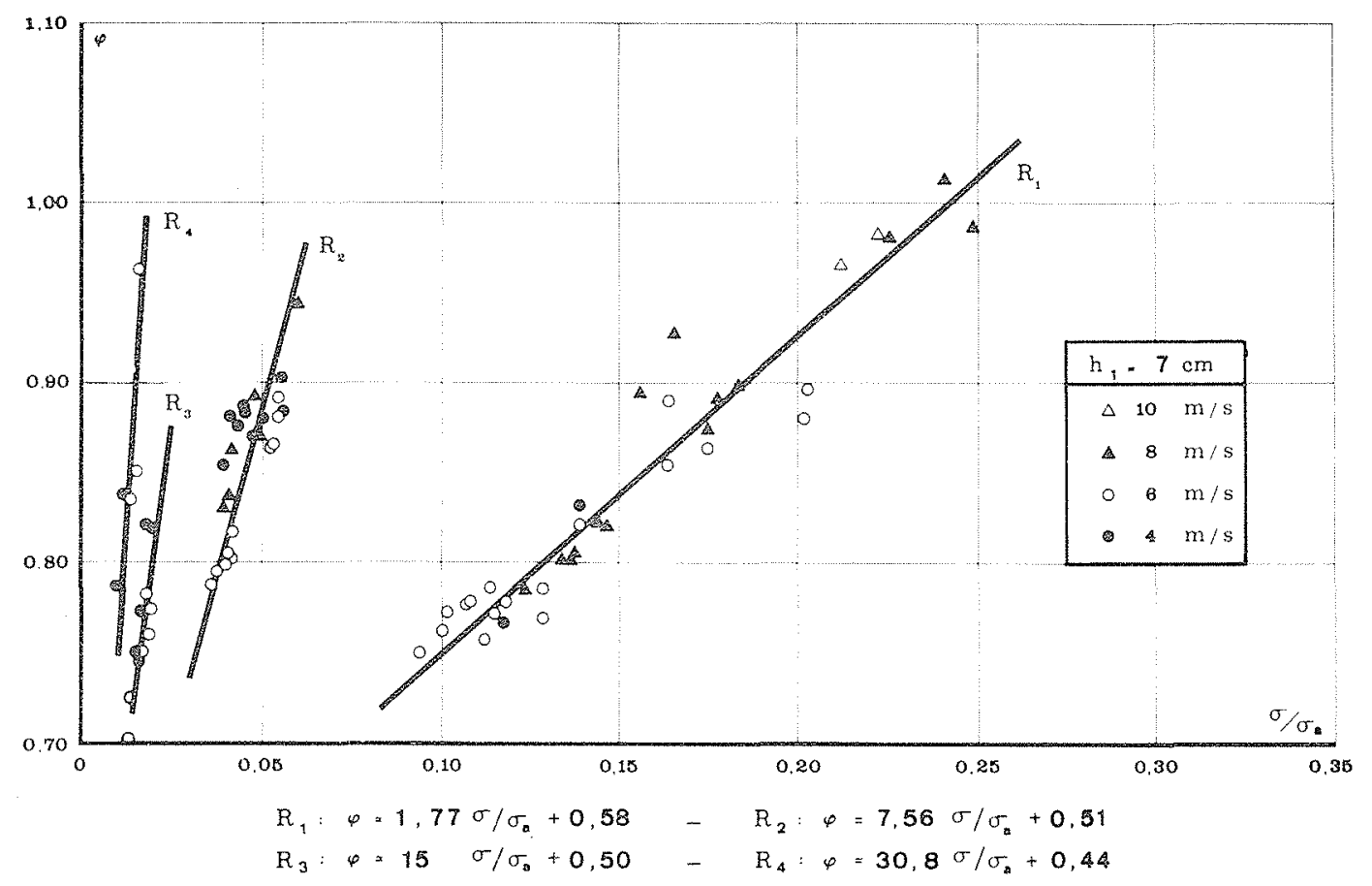

12/ Variations de la fréquence adimensionnelle en fonction de $\sigma / \sigma_{a}$ de l'écoulement aux très faibles vitesses $(\mathrm{V}<2 \mathrm{~m} / \mathrm{s})$, soit légèrement décalés en ordonnées pour des vitesses comprises entre 2 et $5 \mathrm{~m} / \mathrm{s}$. Les photographies de la figure 11 montrent des sillages de cavité correspondant aux schémas de la figure 10. La photographie de la figure 11 a été prise dans le cas d'un écoulement en charge.

Aux vitesses plus grandes, le jet rentrant tend à devenir horizontal. Le paquet d'air évacué de la cavité tend à s'organiser autour de tourbilions aléatoires ou grossièrement alternés. Deux petits tourbillons symétriques, dont l'origine semble être due au jet rentrant, sont souvent présents à l'aval du paquet d'air.

La photographie de la figure 11 b montre que la surface libre du canal n'est pas affectée par les perturbations produites au sein de l'écoulement. II en est ainsi tant que le rapport $h_{1} / \lambda$ est de l'ordre de l'unité. D'un calcul simple [8] il résulte en effet que si la surface libre est soumise à la pression constante $p_{0}$, le taux d'extinction des perturbations de la vitesse et de la pression à une distance verticale y de la cavité est de l'ordre de exp [-2 $\left.\pi \frac{y}{\lambda}\right]$. Ce fait est important: il montre que la présence d'une surface libre dans le voisinage de la cavité $n^{\prime}$ est pas nécessaire à l'existence du phénomène de pulsation.

Des essais, ayant pour but de mettre en évidence la possibilité du phénomène de pulsation des cavités ventilées en domaine confiné, ont été effectués dans la veine à parois solides de la SOGREAH. L'expérience a confirmé les prévisions, basées sur l'analyse des caractères de l'écoulement produit dans un canal ${ }^{*}$. Les phénomènes ont, qualitative- ment, les mêmes caractères. On note cependant que la plage des fréquences est plus restreinte : elle s'étend de 12 à $20 \mathrm{~Hz}$ environ pour les vitesses supérieures à $2 \mathrm{~m} / \mathrm{s}$. L'instabilité de la cavité pour les petits débits d'air, déjà constatée dans le cas du canal, semble renforcée par la présence des parois solides : il nous a été impossible d'obtenir des cavités de longueur inférieure à 9,5 cordes.

\subsection{2 - Fréquence de pulsation}

Utilisant la relation adimensionnelle (e) sour la forme

$$
\varphi=\varphi\left(\frac{h_{1}}{c}, \frac{\bar{l}}{c}, \frac{\sigma}{\sigma_{a}}\right)
$$

on obtient, dans le cas des écoulements à surface libre, un regroupement convenable dans le plan $\left(\sigma / \sigma_{a}, \varphi\right)$ des points expérimentaux correspondant à une immersion relative $\mathrm{h} 1 / \mathrm{c}$. Les figures 12 à 14 montrent cette représentation : pour chacun des régimes $R_{k}$, la variation de $\varphi$ en fonction de $\sigma / \sigma_{a}$ est sensiblement linéaire. La dispersion des points est due aux incertitudes de mesure et ne peut être attribuée à I'influence de la longueur relative $\bar{l} / c$. A une profondeur donnée, on peut donc admettre, dans un régime $R_{k}$ et pour la gamme des essais effectués, une loi de variation du type

$$
\varphi=a_{k} \sigma / \sigma_{a}+b_{k}
$$

Les valeurs des coefficients $a_{k}$ et $b_{k}$, trouvées à partir des moindres carrés, sont données dans les figures 12 à 14 .

L'importance du paramètre $\sigma / \sigma_{a}$ apparait sur les figures 12 à 14 : aux trois profondeurs d'immersion, les régimes $R_{1}$
* En [8], nous montrons que les circonstances expérimentales particulières, notamment la présence d'une surface libre dans le circuit hydraulique, ne permet pas de rendre compte de l'existence du phénomène de pulsation en domaine confiné : l'ordre de grandeur de la fréquence obtenue est environ 30 fois trop petit. De même, nous montrons qu'on ne peut expliquer le phé nomène de pulsation par une interaction entre la cavité et le conduit qui l'alimente. 


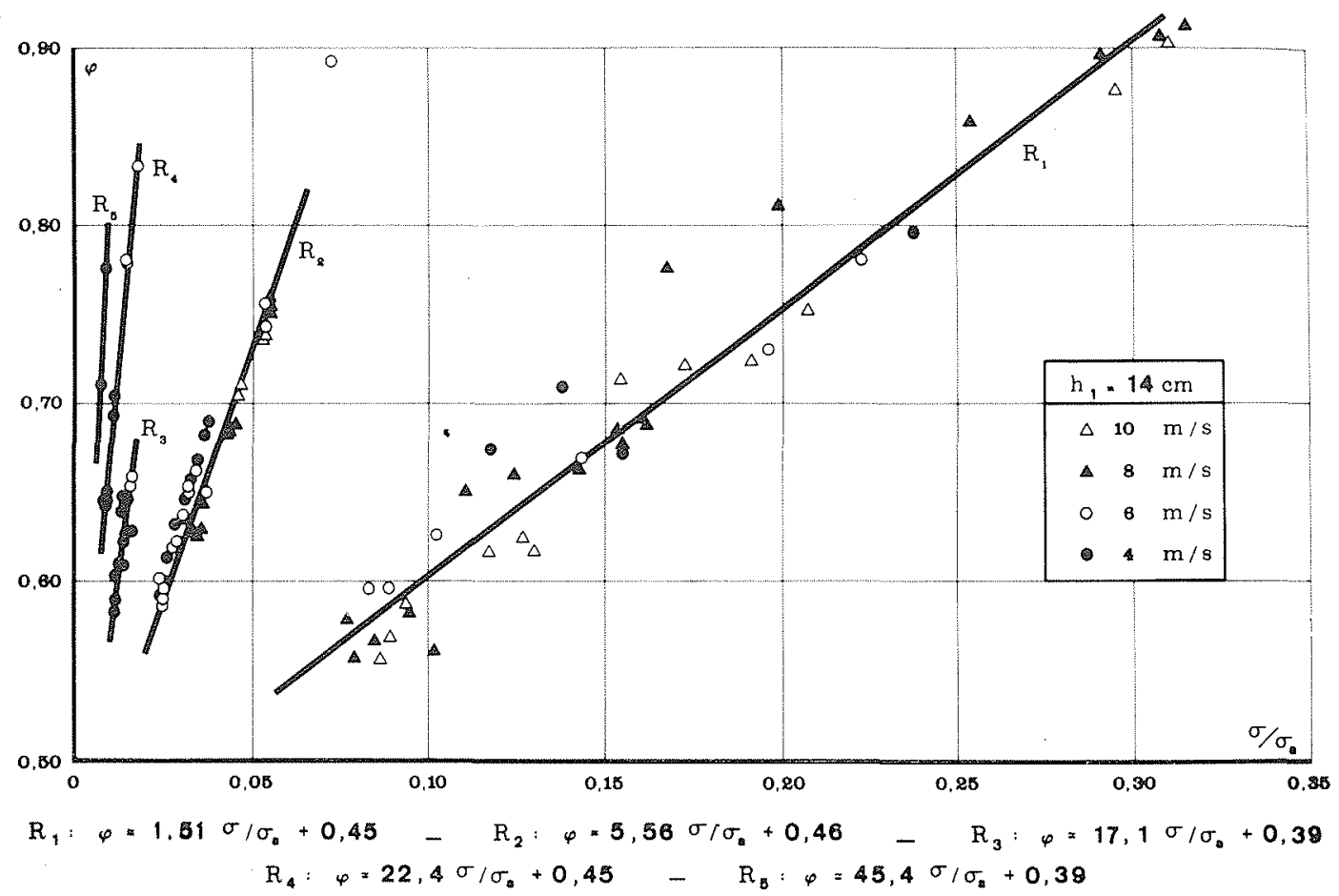

13/ Variations de la fréquence adimensionnelle $\psi$ en fonction de $\sigma / \sigma_{a}$

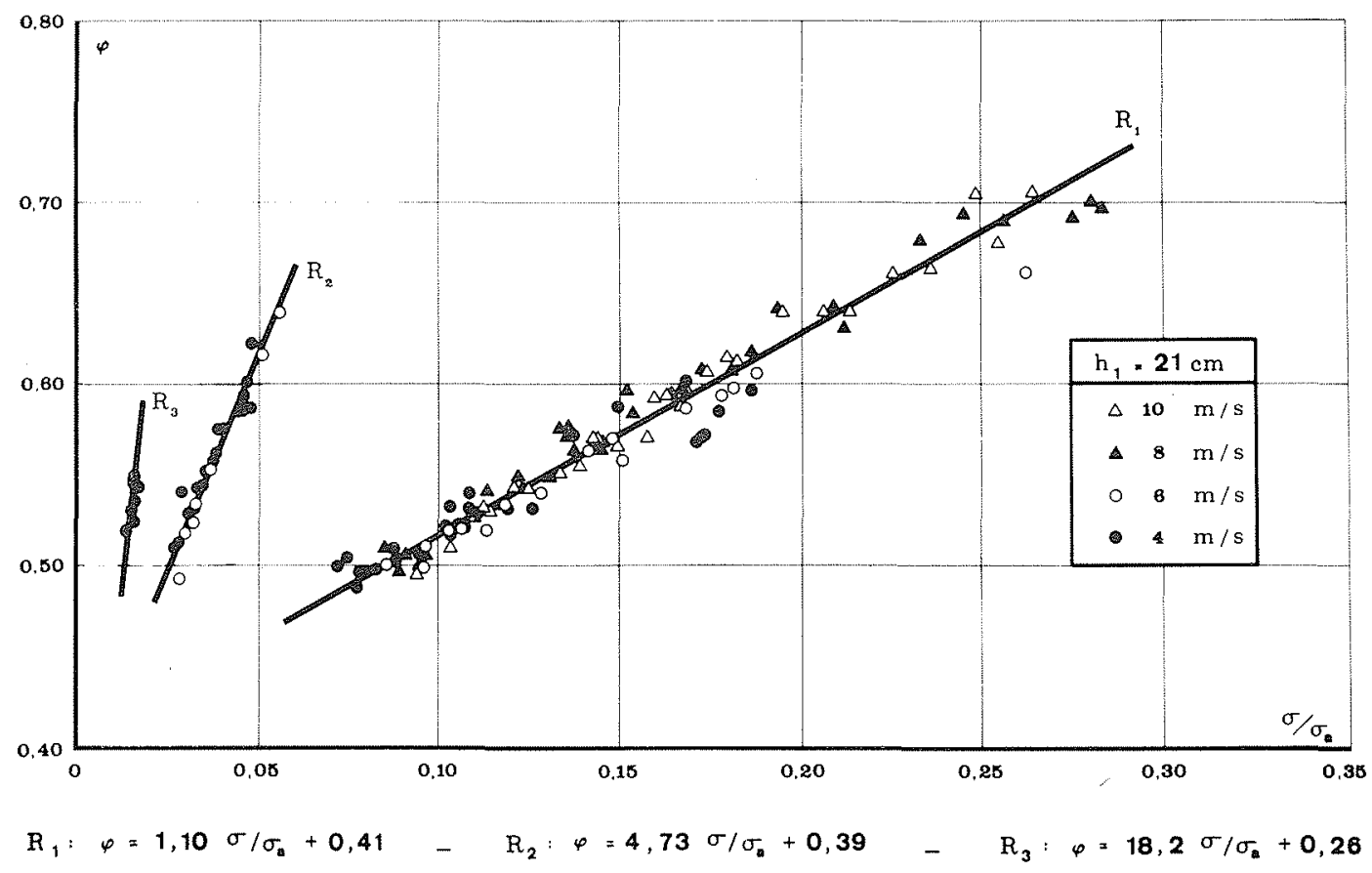

14/ Variations de la fréquence adimensionnelle o en fonction de $\sigma / \sigma_{a}$ 
et $R_{2}$ sont déterminés par des domaines de valeurs séparés de $\sigma / \sigma_{a}$. On a ainsi, sensiblement

$$
\left\{\begin{array}{l}
\text { pour } R_{1}: 0,060<\sigma / \sigma_{a}<0,31 \\
\text { pour } R_{2}: 0,022<\sigma / \sigma_{a}<0,060
\end{array}\right.
$$

Les régimes $R_{3}, R_{4}$ et $R_{5}$ apparaissent dans le domaine de valeurs inférieures à 0,022 . Ces résultats sont notablement différents de ceux de SILBERMAN et SONG, pour qui le rombre $\sigma / \sigma_{v}$ (ou le paramètre $\sigma / \sigma_{a}$ utilisé ici) est pratiquement constant pour un régime donné.

La fréquence adimensionnelle $\varphi$ rapporte la fréquence à la pulsation naturelle $d^{\prime} u n$ piston de masse volumique $\rho$, de hauteur $c$, oscillant au-dessus d'un cylindre de hauteur moyenne $\bar{l}$, qui contient de l'air à la pression $\bar{P}_{\text {air }}$. Son indépendance par rapport à $\bar{\ell} / c$, mise en évidence à partir des résultats expérimentaux, est difficile à justifier sur le plan théorique. Elle est établie cependant dans des conditions suffisamment variées de profondeur d'immersion (relativement à la profondeur de l'écoulement) et de longueur relatives pour qu'il ne soit guère possible de la considérer comme un résultat fortuit. $\|$ en résulte que f est inversement proportionnelle à $\sqrt{\frac{\bar{l}}{\mathrm{c}}}$. La dépendance de $f$ à l'égard de $\bar{P}_{\text {air }}$ est plus complexe ; si cependant on tient compte du fait que les variations de $\varphi$ ne sont pas grandes, il en résulte que $f$ varie principalement comme $\sqrt{\bar{P}_{\text {air }}}$, ce qui confirme les premières indications expérimentales. Ces conclusions tendent à donner quelque consistance à l'image pourtant grossière du système cylindre-piston. Si I'on accepte cette image, on est conduit à attribuer une certaine prépondérance à la compressibilité de l'air présent dans la cavité, inversement proportionnelle à $\overline{\mathrm{P}}_{\mathrm{air}}{ }^{*}$. Onest conduit aussi à admettre que, si le phénomène de pulsationsdes cavités résulte d'échanges d'énergies de natures différentes, ces échanges s'effectuent surtout dans le sens de l'écoulement principal, c'est-à-dire entre la cavité et son sillage. Cette conclusion est renforcée par le fait déjà signalé que, dans beaucoup de cas, la surface libre du canal ne subit pratiquement pas de perturbations verticales ${ }^{* *}$.

\subsection{3. - Longueur d'onde des perturbations des frontières de la cavité}

Les perturbations des surfaces libres de la cavité pulsante prennent la forme d'ondes qui sont transportées, lorsque

\footnotetext{
Si I'on imagine que l'on injecte un liquide à l'arrière du profil et si l'on envisage la possibilité d'un sillage périodique, on est conduit nécessairement à former une relation adimensionnelle où le nombre classique de Strouhal est mis en évidence. Dans le cas des écoulements à cavités ventilées il est impossible de corréler les points expérimentaux à partir du nombre de Strouhal ; cela peut être vérifié facilement à partir de la figure 9 ou des figures 12 à 14 . II en résulte que dans ce cas, la compressi. bilité de l'air tend à masquer les effets propres de son inertie, si celle-ci joue un rôle éventuel dans le mécanisme de pulsation.

* En milieu confiné, le regroupement des points expérimentaux dans le plan $\left(\sigma / \sigma_{a}, \varphi\right)$ ne peut se faire qu'à longueur de cavité ou à $\sigma$ constant; la tendancevgénérale est alors la méme qu'en écoulement à surface libre. La dépendance de $\varphi$ à l'égard de $\sigma$ qui traduit l'influence des parois solides, empêche d'écrire la relation (3) dans le cas des écoulements confinés.
}

les forces de pesanteur sont négligées, à la vitesse $U_{C}$ de l'écoulement principal sur ces surfaces. Pour une fréquence $f$ du phénomène périodique, la longueur d'onde est :

$$
\lambda=\frac{U_{c}}{f}=\frac{V \sqrt{1+\sigma}}{f} \simeq \frac{V}{f}\left(1+\frac{\sigma}{2}\right)
$$

La comparaison systématique de l'une des grandeurs caractéristiques de la cavité, $\ell_{m}, \bar{l}$ et $\ell_{M}$, avec la longueur $k \lambda$ (dans un régime $R_{k}$ ) nous a donné les résultats suivants.

a) Lorsque le profil est placé au milieu du canal $\left(\mathrm{h}_{1}=14 \mathrm{~cm}\right)$, la moyenne du rapport $\ell_{\mathrm{m}} / \mathrm{k} \lambda$ est égal à 0,999 , avec un écart-type de 0,044 (moyenne établie sur 109 points). Lorsque le profil est placé au milieu de la veine à parois solides, les valèurs du rapport et de l'écart-type sont, pour 39 points étudiés, de 1,013 et 0,060 .

b) Lorsque le profil est placé près de la surface libre $\left(h_{1}=7 \mathrm{~cm}\right)$, les valeurs moyennes de $l_{m} / k \lambda$ et $\bar{l} / k \lambda$ sont à peu près également distantes de l'unité : 0,954 et 1,043 ; les écarts-types sont assez grands : 0,066 et 0,073 .

c) Pour la plus grande profondeur d'immersion, c'est le rapport $\ell_{M} / k \lambda$ qui est en moyenne le plus proche de l'unité. Sa valeur, pour 139 points de mesure, est de 1,033.

Il semble donc difficile de conclure en général à une loi unique reliant une des grandeurs caractéristiques de la cavité à la longueur d'onde des perturbations. Si on considère seulement le cas où le profil est placé à mi-hauteur du canal, qui est aussi celui où la mesure de $\ell_{m}$ a été faite avec le plus de précision, on peut adopter dans ce cas la loi

(5) $\ell_{m}=k \lambda$

Cette loi signifie que l'instant où les perturbations dirigées vers l'intérieur de la cavité se rejoignent à l'arrière de celle-ci et provoquent ainsi l'évacuation d'un paquet d'air,est aussi l'instant où elles prennent naissance aux bords de fuite du profil.

\subsection{4. - Fluctuations de la pression}

La mesure des fluctuations de la pression dans la cavité et en quelques points de l'écoulement permet de tirer les conclusions suivantes:

a) Les fluctuations de la pression dans la cavité sont uniformes, en phase et en amplitude. Ce fait confirme le rôle négligeable de l'inertie de l'air qui s'écoule dans la cavité, par rapport à sa compressibilité.

b) La cavité impose la phase des fluctuations de la pression dans toute la zone proche de l'écoulement. L'am. plitude des fluctuations décroit sensiblement au voisinage de la surface libre, suivant la loi exponentielle indiquée plus haut (par. 2.3.1.).

c) Le minimum de la pression se produit à peu près à l'instant où la cavité atteint presque simultanément sa longueur minimale et sa longueur maximale ; le déphasage entre ces deux instants est compris entre $1 / 24$ e et $1 / 10$ e de période environ. 


\subsection{5. - Cinématique des cavités ventilées}

La figure 15 représente les différentes étapes, obtenues par un procédé de chrono-enregistrement donnant une bonne précision [10], de l'évolution d'une cavité à une longueur d'onde au cours d'une periode dans les circonstances expérimentales suivantes $: \mathrm{h}_{1}=14 \mathrm{~cm}, \mathrm{~V}=4 \mathrm{~m} / \mathrm{s}, \sigma=0,111, \sigma / \sigma_{\mathrm{a}}$ $=0,137$. La fréquence était alors de $11,1 \mathrm{~Hz}$ et la longueur de la cavité variait entre 36 et $55 \mathrm{~cm}$. Les différentes étapes sont repérées par leur déphasage $\phi$, exprimé en fraction de période, par rapport à la phase du dispositif expérimental, utilisé comme repère du temps. Le minimum de la pression $P_{C}$ correspond à peu près à $\phi=0,1$.

On relève sur la figure 15 les faits principaux suivants : la naissance, aux bords de fuite du profil, des perturbations des frontières de la cavité, dirigée vers son intérieur, à l'instant où le paquet d'air s'en détache ; la propagation quasi- symétrique de ces perturbations à une vitesse pratiquement égale à la vitesse $U_{c}$ des particules dans l'écoulement moyen; leur amplification vers l'aval ; la progression de l'arrière de la cavité à une vitesse dont la valeur moyenne est inférieure à $U_{c}$; cette vitesse, d'abord presque nulle, croît en même temps que la longueur de la cavité ; la formation des paquets d'air par la jonction des perturbations développées sur les frontières de la cavité ; enfin la progression des paquets d'air à une vitesse légèrement inférieure à la vitesse d'entrée $V$ de l'eau. La dissymétrie de la cavité et des paquets d'air par rapport à la ligne moyenne de l'écoulement est une conséquence de la gravité qui dévie le jet rentrant vers le haut.

Dans les circonstances expérimentales de la figure 15 , les variations relatives $\Delta \sigma / \sigma$ étaient d'environ $1 / 10$, avec $\triangle \ell / \bar{l} \simeq 1 / 2$. Dans une théorie comme celle de HSU et CHEN, qui considère la masse d'air contenue dans la cavité

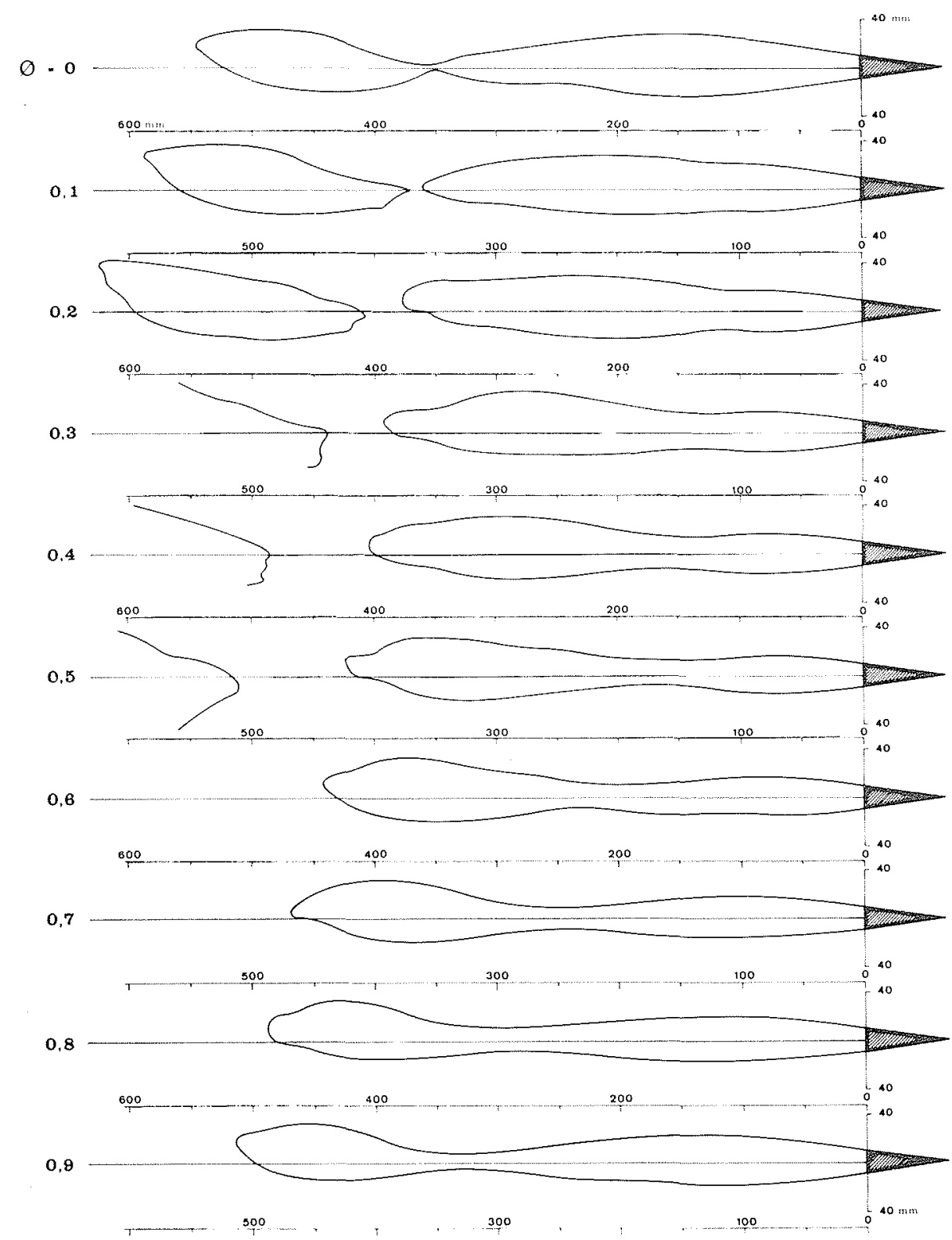

15/ Evolution d'une cavité à longueur d'onde au cours d'une période 
comme constante, le rapport $\Delta \& / \bar{l}$ est de l'ordre de $(\triangle \sigma / \sigma)^{2}$. Cette comparaison permet d'évaluer l'influence, déjà manifeste dans les schémas de la figure 15 , de l'apport d'air à la base du profil et de son mode d'évacuation à l'arrière de la cavité.

\section{3 - CARACTERES PHYSIQUES DE LA PULSA- TION DES CAVITÉS VENTILEEES}

L'examen des principales difficultés de la théorie des écoulements supercavitants non permanents et des solutions qui leur ont été données pour expliquer la pulsation des cavités ventilées nous permettra de dégager les principaux caractères physiques de ce phénomène et de proposer un mécanisme qualitatif éclairant plusieurs de ses aspects.

La première difficulté théorique est relative à la dépendance de l'écoulement à surface libre non permanent par rapport à son histoire antérieure [11]. Cette dépendance disparaît lorsqu'on suppose l'écoulement périodique. Dans le cas des écoulements à cavités ventilées, elle n'apparaît donc que si l'on considère les phases transitoires de l'écoulement : l'apparition des cavités, la croissance ou la diminution de leur volume moyen consécutives, par exemple, à des variations du débit d'air injecté. Cette dépendance, qui constitue de soi une difficulté importante pour le traitement théorique des écoulements non permanents, permet donc ici de comprendre l'aspect, à première vue singulier, de la relation entre le débit d'air et le nombre $\sigma$ (fig. 8 ) : si la réponse actuelle du système formé par l'écoulement dépend non seulement des grandeurs d'entrée mais aussi de I'histoire antérieure, l'existence, pour une même valeur des grandeurs d'entrée, de plusieurs points de fonctionnement autour desquels le régime périodique peut s'établir, est admissible.

La seconde difficulté théorique concerne la singularité de la pression qui apparaît en écoulement plan infini ou en écoulement limité par des parois solides lorsqu'on prend en compte les variations de volume d'une cavité finie située au sein de l'écoulement. A grande distance $r$ de la cavité, les variations de volume se traduisent, pour le potentiel de perturbation, par un terme équivalent à un potentiel de source soit, en domaine plan illimité, par

$$
\varphi(r, t) \simeq \frac{q(t)}{2 \pi} \log r, r \rightarrow \infty
$$

expression dans laquelle $q(t)$ désigne le débit variable de la source. L'équation de Bernoulli généralisée montre alors qu'au voisinage de l'infini la pression se comporte comme $\partial \varphi / \partial t$, d'où une singularité logarithmique. En écoulement unidimensionnel, la singularité est plus forte encore, puisque I'on a dans ce cas $: \partial \varphi / \partial \mathrm{t} \simeq \mathrm{r}$.

Au lieu de l'écoulement autour d'un profil et d'une cavité, on peut considérer simplement une bulle circulaire contenant une certaine masse de gaz au sein d'un liquide au repos à l'infini. La singularité précédente de la pression, qui est due à une décroissance trop lente de la vitesse à l'infini et par suite à une valeur infinie de l'énergie cinétique, signifie qu'il est impossible d'envisager dans ce schéma une pulsation de la bulle par échange d'énergie cinétique du liquide et d'énergie élastique du gaz autour d'une position d'équilibre stable. En d'autres termes, les simples oscillations d'un système conservatif sont impossibles en milieu plan infini ou en domaine unidimensionnel confiné entre des parois solides.

SONG [2], d'une part, HSU et CHEN [3], d'autre part, tout en adoptant le schéma du système conservatif à masse d'air constante, ont tourné la difficulté précédente de deux manières différentes. Le premier a adapté le modèle de la bulle circulaire au cas des cavités ventilées : la présence d'au moins une surface libre extérieure dans le voisinage de la cavité est alors nécessaire à l'existence de la pulsation. Les seconds auteurs ont considéré une cavité pulsant au sein d'un domaine de liquide infini. Au voisinage de la cavité, le liquide est considéré comme incompressible ; dans le domaine lointain au contraire, sa compressibilité est prise en compte, ce qui permet d'éviter la singularité de la pression. La même méthode de raccordement a été utilisée par BENJAMIN [12] : cet auteur invoque, pour le domaine lointain, la nature nécessairement tri-dimensionnelle de l'écoulement qui fait aussi disparaître la singularité de la pression.

Ces solutions, bien que formellement irréprochables, ne sont pas entièrement convaincantes en ce que, outre certaines divergences avec la réalité physique [8], elles font appel à des mécanismes extérieurs pour établir la possibilité de la pulsation, ce qui revient à interdire cette possibilité dans le cas du fluide strictement incompressible et du domaine plan infini. Le fait que la pulsation des cavités se produise en écoulement confiné entre des parois solides pour lequel la singularité de la pression est en principe plus forte qu'en milieu infini, sans que les circonstances expérimentales particulières, notamment la présence d'une surface libre loin à l'aval de la section d'essai, puissent rendre compte de l'ordre de grandeur des fréquences de pulsation obtenues, nous incite à admettre la possibilité des pulsations même en milieu plan infini.

WOODS [4] a ouvert une voie en ce sens en considérant d'emblée, à l'arrière d'un profil symétrique placé en milieu illimité, une cavité suivie d'un sillage infini, et en imposant au volume total de la cavité et de son sillage d'être constant, ce qui supprime la singularité de la pression à l'infini. Dans le modèle de WOODS, l'écoulement du gaz qui remplit la cavité n'est pas pris en compte; mais les fréquences de pulsation sont obtenues comme valeurs propres d'une relation empirique qui traduit la réponse de l'écoulement à une variation de pression dans la cavité. Ces fréquences propres, définies dans nos notations par:

$$
\omega_{k}=\frac{\pi f \ell_{m}}{v \sqrt{1+\sigma}}=\frac{\pi \ell_{m}}{\lambda}
$$

ont pour valeur approximative :

$$
\omega_{k}=1,97+(k-1) \pi
$$

Selon nos résultats expérimentaux, lorsque la loi (5) : $\ell_{\mathrm{m}}=\mathrm{k} \lambda$ est acceptable, la valeur correspondante des pulsations adimensionnelles est : $\omega_{k}=k \pi$. Cette divergence 
n'est pas très grave puisqu'aussi bien la relation (5), qui ne traduit qu'une relation de phase entre l'instant où les perturbations naissent à la base du profil et celui où elles se rejoignent à l'arrière de la cavité, n'est pas vérifiée dans toutes les circonstances expérimentales. Plus important nous semble l'aspect positif de la condition de WOODS sur la constance du volume de la cavité et de son sillage, qui fournit la possibilité d'un mécanisme de pulsation indépendant des frontières de l'écoulement. Les variations compensées des volumes de la cavité et du sillage, qui résultent de cette condition, peuvent être interprétées en termes d'échanges de masse d'un fluide qui remplirait la cavité et par conséquent en termes d'échanges d'énergie entre la cavité et le sillage : nos résultats d'essais tendent aussi à la conclusion que les échanges d'énergie s'effectuent principalement suivant la direction de l'écoulement général. Enfin, il est remarquable que le système considéré par WOODS n'est pas conservatif ; ce caractère ressort aussi de la description physique de l'écoulement à cavités ventilées : l'écoulement emporte à tout instant vers l'aval et dissipe l'énergie cinétique fluctuante reçue au voisinage de la cavité. Le problème consiste alors à identifier la source de l'énergie fluctuante et à préciser les principales modalités du transfert d'énergie.

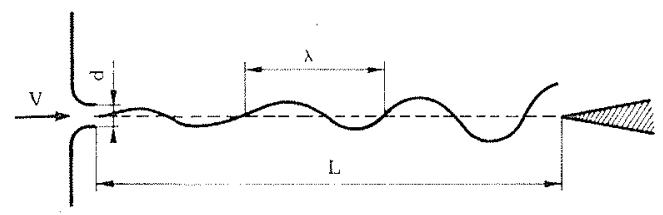

161

NAUDASCHER [5], dans un article synthétique consacré aux écoulements auto-oscillants, a décrit l'un des plus simples d'entre eux, le "son de biseau" (traduction reçue du terme anglais "edgetone") et a étudié l'influence d'un résonateur sur un tel écoulement. Le son de biseau est produit par un jet libre plan qui frappe un obstacle anguleux placé dans son plan moyen (fig. 16). Aux petits nombres de Reynolds, l'écoulement peut prendre un aspect périodique de fréquence $f$; la ligne moyenne du jet prend alors une forme ondulée et il apparaît une double file de tourbillons alternés. La fréquence varie de manière continue pour des petites variations de $V$ et $L$; elle peut aussi subir des sauts lorsque le nombre de tourbillons sur une file augmente d'une unité. On observe ainsi des régimes d'écoulement caractérisés par le nombre de tourbillons dans une file, le passage d'un régime à l'autre se faisant pour des valeurs limites différentes de $V$ ou $L$ suivant que le nombre de tourbillons augmente ou diminue : les diagrammes $S(L / d)$ ou $S\left(R_{e}\right)$ (S désigne le nombre de Strouhal $f d / V$, $R_{e}$ le nombre de Reynolds $V d / \nu$ ) font apparaître des boucles d'hystérésis. On retrouve là un caractère analogue à celui des pulsations de cavités.

Le mécanisme d'oscillation du "son de biseau" requiert d'abord l'instabilité naturelle du jet libre laminaire pour une certaine bande de fréquence et ensuite une loi de phase par laquelle la croissance d'une seule fréquence privilégiée est favorisée par la réaction du coin symétrique. Cette loi de phase est décrite par la relation :

$$
L=\left(k+\frac{1}{4}\right) \lambda
$$

qui est vérifiée aussi dans d'autres configurations d'écoulements auto-oscillants. Cette loi de phase et la largeur finie de la bande d'instabilité du jet expliquent alors le caractère hystérétique de la relation $S\left(R_{e}\right)$. Lorsqu'un résonateur est placé au voisinage du son de biseau, le mécanisme d'auto-oscillation $n$ 'est pas fondamentalement modifié : le résonateur a pour effet majeur d'élargir le domaine d'ins. tabilité du jet lorsque la fréquence naturelle du son de biseau est voisine d'une de ses fréquences propres, de sorte que la réponse en fréquence du système oscillant revêt une forme différente.

NAUDASCHER suggère que le mécanisme de pulsation des cavités ventilées soit identifié à celui du son de biseau avec résonateur, organe dont le rôle serait tenu par la cavité elle-même. S'il existe effectivement des analogies formelles entre les deux types d'écoulements, on doit insister cependant sur quelques différences significatives qui rendent difficile cette assimilation :

a. Dans le son de biseau les frontières et les conditions d'écoulement moyen sont symétriques tandis que les perturbations sont anti-symétriques. Au contraire, dans le cas des cavités ventilées, la dissymétrie imposée par les frontières de l'écoulement ou par le champ de gravité n'affecte pas profondément l'écoulement fluctuant qui tend à conserver la symétrie par rapport à l'axe moyen du profil.

b. Alors que dans le son de biseau la distance $L$ entre l'orifice du jet et le sommet du coin est fixée, il n'existe pas, dans le cas des cavités ventilées, de longueur de référence pour l'écoulement moyen. La variation continue du débit s'y traduit, lors des changements de régime, par des discontinuités des grandeurs moyennes $\sigma, \bar{l}$ et $l_{m}$ tandis que les grandeurs caractéristiques de l'écoulement fluctuant telles que la longueur d'onde $\lambda$ et la fréquence $f$ ne subissent que des variations très petites. II en résulte que la loi (5) : $\ell_{m}=k$, dans les cas où elle est vérifiée, ne joue de rôle déterminant ni dans la relation de phase du mécanisme de contre-réaction ni dans le passage d'un régime à un autre.

c. Le nombre de Strouhal exprime sous forme globale le transfert d'énergie entre l'écoulement moyen et l'écoulement fluctuant. Toutefois cette interprétation n'est possible qu'en raison du caractère monophasique de l'écoulement constitué par le son de biseau. Le caractère diphasique de l'écoulement à cavités ventilées se traduit par la relation $\varphi\left(\sigma / \sigma_{a}\right) ;$ l'impossibilité de corréler dans ce cas les résultats expérimentaux à partir du nombre de Strouhal traduit l'influence prépondérante de la compressibilité de l'air. L'énergie fluctuante du liquide doit donc trouver sa source principale dans l'apport du gaz compressible. La petite valeur du transfert d'énergie entre l'écoulement moyen et l'écoulement fluctuant du liquide est d'ailleurs une conséquence naturelle de la géométrie des écoulements que nous considérons : la courbure très petite des lignes de courant dans l'écoulement moyen implique des gradients de vitesse moyenne presque négligeables. 
$* *$

Les caractères principaux du phénomène de pulsation des cavités ventilées, livrés directement par l'expérience ou dégagés à la suite de l'examen des modèles théoriques du phénomène, peuvent être résumés maintenant.

a. En l'absence de longueur de référence dans l'écoulement moyen, la réponse moyenne et la réponse fluctuante de l'écoulement dépendent simultanément des grandeurs permanentes d'entrée.

b. Par suite la relation éventuelle entre une dimension caractéristique de la cavité et la longueur d'onde des perturbations ne joue aucun rôle déterminant. La seule rela. tion de phase importante est celle qui lie la pression dans la cavité à la géométrie de la cavité : la pression passe par son minimum à peu près à l'instant où la cavité atteint simultanément (ou presque simultanément) sa longueur minimale et sa longueur maximale.

c. La symétrie des perturbations des frontières de la cavité conduit à attribuer un rôle important à la pression dans la naissance de ces perturbations.

d. Le système dynamique formé par le liquide et le gaz n'est pas conservatif. L'énergie fluctuante du liquide a sa source principale dans l'apport du gaz compressible.

e. Les échanges d'énergie se font principalement suivant la direction de l'écoulement principal. Cette conclusion de l'expérience s'accorde bien avec l'hypothèse de WOODS sur la constance du volume total de la cavité et du sillage, seule voie théorique offrant la possibilité d'un mécanisme de pulsation indépendant des frontières de l'écoulement.

L'expérience montre encore un autre caractère important des écoulements à cavités ventilées : la pulsation ne se produit que si l'écoulement est bi-dimensionnel, plan ou de révolution. Les effets tridimensionnels dus à la gravité ou à l'envergure finie d'une aile tendent à diminuer I'intensité des pulsations ou à les faire disparaître. II est remarquable que le caractère bi-dimensionnel entraîne l'existence d'une zone de surpression à l'arrière de la cavité et l'amorce d'un jet rentrant. En régime permanent, sensiblement réalisé en cavitation de vapeur, la double condition d'uniformité de la pression dans la cavité et de valeur minimale de cette pression par rapport au voisinage impose aux frontières de la cavité une courbure partout dirigée vers l'intérieur de la cavité, ce qui provoque la formation du jet rentrant et de la zone de surpression. Cette double condition est encore réalisée lorsque l'air est injecté à la base du profil de sorte que son écoulement continu à l aval de la cavité est impossible. II apparaît ainsi que la condition de blocage de l'air à l'intérieur d'une cavité fermée est essentielle au phénomène de pulsation : c'est cette condition qui permet le transfert d'énergie entre l'écoulement d'air et l'écoulement de liquide.

L'importance de la zone arrière de la cavité en écoulement plan peut être mise en évidence par l'expérience suivante. Deux plaques minces parallèles horizontales, de longueur $300 \mathrm{~mm}$, distantes de $24 \mathrm{~mm}$, sont placées sur toute la largeur du canal à l'arrière de la cavité. Lorsque le plan moyen des deux plaques est situé à hauteur du bord d'attaque du profil, la cavité prend, pour un débit d'air convenable, la forme stable de la figure 17. La fonction

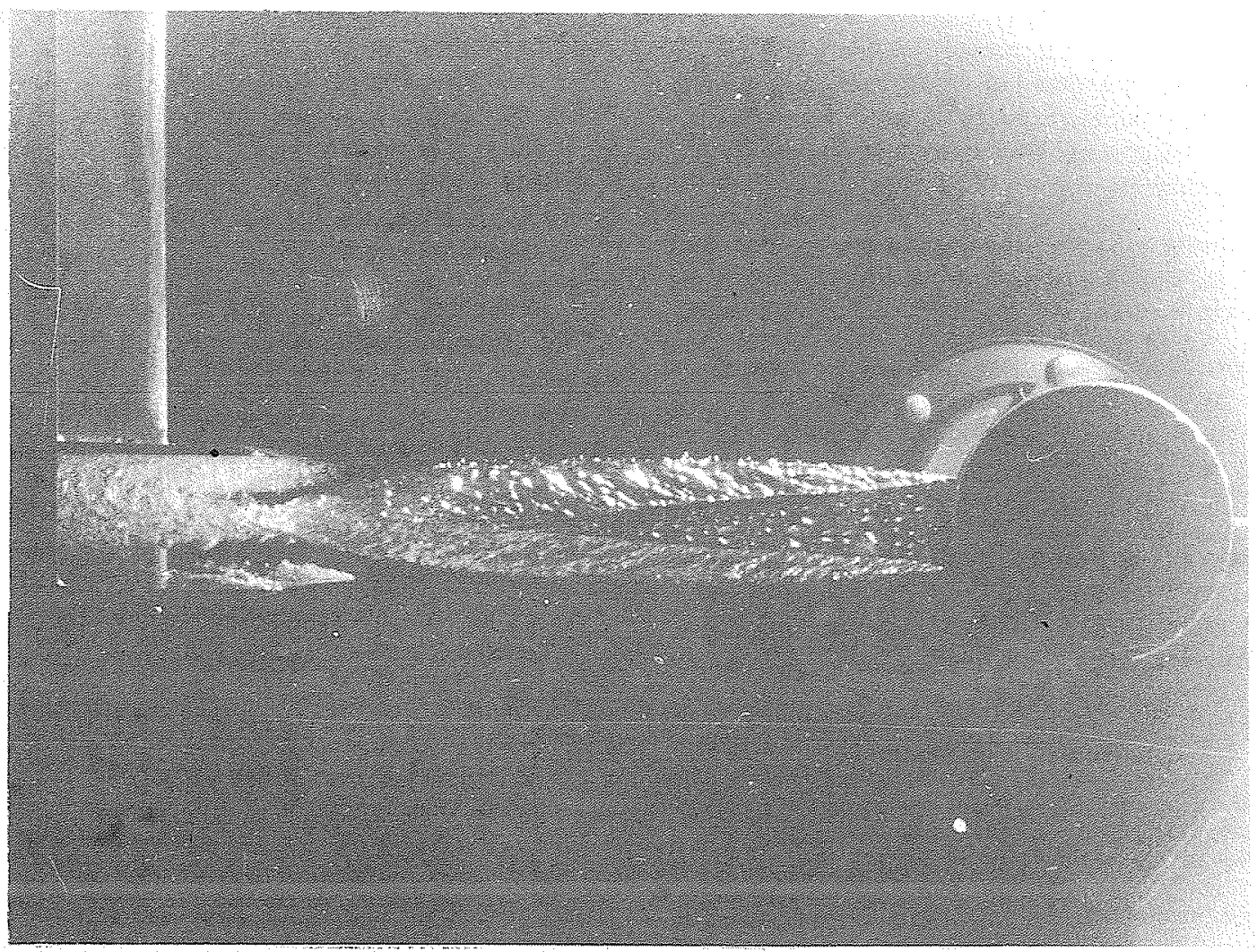




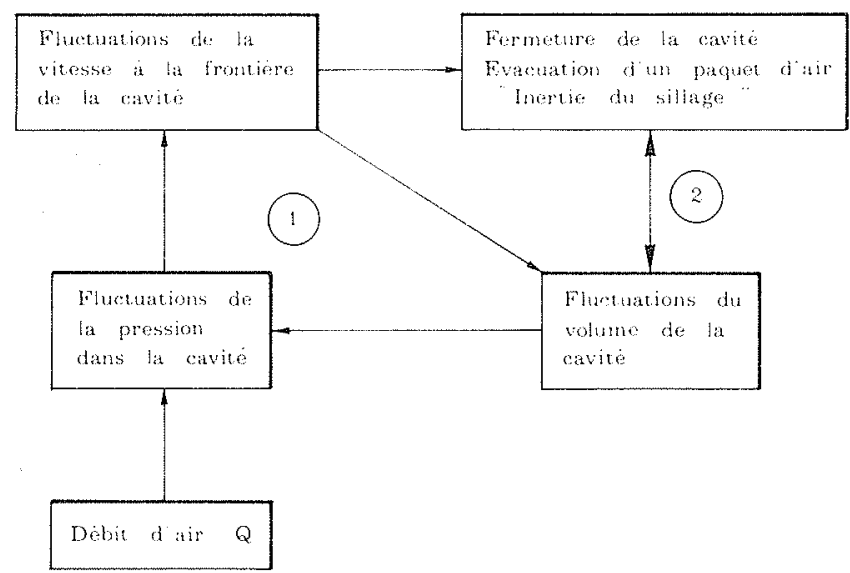

181

des deux plaques consiste à créer à l'intérieur du liquide des zones de dépression qui permettent un changement du sens de la courbure des frontières de la cavité : la zone de surpression est supprimée, l'air s'écoule alors d'une manière continue, la pression dans la cavité est constante et le phénomène de pulsation ne se produit pas. Les ondes qui se développent sur les frontières d'une cavité pulsante, et qui constituent son aspect le plus spectaculaire, n'apparaissent pas dans le cas présent, bien que les mécanismes d'instabilité nécessaires à leur amplification existent en fait : leur naissance aux bords de fuite du profil nécessite l'excitation par les variations de la pression dans la cavité et donc la condition de blocage de l'air dans la cavité*.

Si la condition de blocage explique le caractère discon. tinu de l'écoulement de l'air à l'aval de la cavité, elle ne permet pas à elle seule de rendre compte de la périodicité de l'écoulement. Cet aspect essentiel des écoulements à cavités ventilés implique un mécanisme da contrôle par lequel les principales grandeurs en leu s'ordonnent suivant une loi de phase et d'amplituda convenable. Nous tentons l'examen de ce mécanisms en considérant la châne des interactions suivantes :

A. Les variations de la pression dans la cavité entrainent la naissance et le déveleppement des fluctuations de vitasse, notamment transversales, sur les frontières de la cavité.

B. Les fluctuations de vitesse ont pour conséquence d'une part la variation du volume de la cavité et d'autre part la fermeture de la cavlté, a'estràrdire l'apparition d'une nouvelle zone de surpression et l'śvacuation d'un nouveau paquet d'air.

C. La croissance du volume de la cavité, caractériste principalement par l'augmentation de la longueur $\ell$, a pour effet, conjointement avec I'injection d'air, de faire varler la pression dans la cavité.

\footnotetext{
* La valour de la surprossion d'arricre d'une cavito de vapour a été trouvée assez petlto, par sulte do l'émission des bulles do vapeur dans cetto zono (par, 2.2.). II ne nous a pas áté possiblo de mo surer la valour maximale do cetto surpresalon dans lo cas des

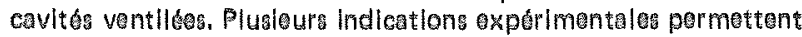
cepondant d'estimer qu'olle est nottement plus Importanto done co cas:
}

D. La variation du volume de la cavité ne peut être cependant considérée indépendamment de l'ensemble de l'écoulement : un de ses effets majeurs est de déplacer vers l'aval la zone de fermeture de la cavité et le paquet d'air. Ce phénomène, qui traduit le travail des forces de pression dans la cavité contre l'inertie du sillage, constitue un des aspects essentiels du mécanisme de régulation. II doit être soumis, suivant les indications de l'expérience, à la condition de WOODS sur la constance du volume de la cavité et du sillage.

Ces interactions sont résumées dans le schéma de la figure 18. Dans ce schéma, le débit d'air joue un rôle essentiel non seulement parce que l'écoulement en masse de l'air est pris en compte, mais encore parce que cet écoulement fournit, pour le système non conservatif que nous considérons, l'énergie indispensable à l'entretien des fluctuations.

La traduction quantitative du schéma de la figure 18 devrait en principe fournir les relations nécessaires pour déterminer toutes les grandeurs prises en compte dans la description schématique de la cavité et du sillage. En l'état actuel, cette traduction est difficile, soit à cause de la complexité des phénomènes à l'aval de la cavité, soit parce que certaines grandeurs (surpression dans la zone de fermeture, masse virtuelle associée au paquet d'air) n'ont pu être mesurées ou calculées. Quelques indications de type qualitatif suffiront ici pour metre en évidence la compatibilité des sens de variation de quelques grandeurs envisagées. La condition de WOODS doit être étendue seulement au domaine du sillage proche de la cavité, domaine limité à une ou deux longueurs d'onde : au-delà, les paquets d'air $n^{\prime}$ vevoluent pratiquement plus. Cette condition est alors compatible avec le fait que la pression dans la cavité est minimale à l'instant du détachement des paquets d'air. Le volume de ceux-ci doit done diminuer lorsqu'ils sont trans. portés vers l'aval ; la masse virtuelle associée aux paquets d'air diminuant, ils subissent une accélération pour rejoin. dre le domaine lointain du sillage. D'autre part, dans le même temps, la surpression \& l'arrière da la eavité, maxi= malo 首 l'instant du dêtachement des paquets d'air, doit décrôtre lorsque catte zone rejoint le sillage lointain ; cetto décrolssance ast complatible avec l'augmentation do la vitasse de la partie arriere de la cavitá.

En rêsumé, la pulsațon des eavités ost dua d'abord au fait que la cavitá est fermée. Lo mécanisme régulateur des pulsations résulte d'une part de l'inertie du demalne du sillago proche de la eaviț́, reeréé 首 chaque période par la jonction des perturbations des frontieres do la cavité, ot d'autre part des forces de pression variables angendrées par les varlations du volume de la cavitis at par l'apport d'air à la base du profill, forces dont la rôla consiste à accélérer la masse liquide constituant lo domaine proche at la repousser dans lo domeine lointain où olle atteint un état d'équilibre dynamique.

La surprossion dans la zone arrièra de la cavité, par laquelle la condition de blocage de l'alr est assurée, permet de comprendre aussi daux aspects particuliers do l'écoulo: ment 首 cevitís ventilǵas. En premier lieu, l'instabilití de l'écoulement, qul se manifeste par un allongement brutal de la cavití lorsqu'on Injecte une patite quantité d'air a la base du profil, peut etro comprise assez facilement si l'on admet qu'une des longueurs caractéristiques de la cavi- 
té, par exemple sa longueur minimale, doit être de l'ordre de grandeur d'au moins une longueur d'onde pour que le mécanisme de contrôle des pulsations puisse s'établir. On trouve alors que l'instabilité est plus forte lorsque la pression de référence est plus petite $\left(\sigma_{\mathrm{V}}\right.$ petit) ; c'est bien ce que l'expérience fait apparaître. En second lieu, il semble possible de rendre compte des changements de régime en considérant simultanément la surpression $\Delta p_{f}$ de la zone de fermerure et la pression dans la cavité. La première tend à stabiliser l'écoulement dans un régime par rapport au régime d'ordre supérieur et à favoriser le passage au régime d'ordre inférieur ; la seconde a le rôle opposé. Elles varient en sens contraire lorsqu'on augmente le débit d'air : la première décroit, la seconde croît. Le passage à un régime supérieur doit alors correspondre à une valeur moyenne insurfisante du rapport $\Delta p_{f} \bar{p}_{C}$ tandis que le passage au régime inférieur doit correspondre à une valeur excessive de ce rapport.

Si cette analyse est correcte, le changement de régime traduit une insuffisance du mécanisme de contrôle de la pulsation, c'est-à-dire une instabilité globale de l'écoulement. Lallure en boucles d'hystérésis du diagramme $\mathrm{Q}(\sigma)$ est alors liée a lexistence de domaines finis de valeurs du rapport $\Delta p$. $\sqrt{p_{0}}$ qui assurent la stablité de l'ćcoulement dans chacun des régimes de pulsation.

Cét article résume les résuthats expérimentaux au ont tait lobict d'un ménoire de these [8]. Oueloues résultats ont téte publiés en [13]. L'étude a été hinancée par la Direction des Recherches et Moyens d'Essais lconventions 118 . 60 at $441.69 \%$.

\section{REFERENGES}

1. SILBERMAN E. - SONG C.S. "nstability of ventilated cavities".

J. of Ship Res. 5-1 (1961), p. 1333.

2. SONG C.S. "Pu/sation of ventilated cavilies". J. of Ship Res. 5-4 (1962), p. $1-20$.
3. HSU C.C. - CHEN C.F. "On the pulsation of finite ventilated cavities".

Hydronautics, Techn. Rept 115-4 (1962).

4. WOODS L.C. "On the instability of ventilated cavi. ties".

J. of Fluid Mech. 26 (1966), p. 437-457.

5. NAUDASCHER E. "From flow instability to flowinduced excitation".

A.S.C.E. J. of the Hydraulic Division (June 1967), p. $15-40$.

6. DODU J. - DUPORT J. - MICHEL J.M. "Le tunnel hydrodynamique de l'Université de Grenoble". La Houille Blanche (1968), p. 697-702.

7. BRENNEN C. "The dynamic balances of dissolved air and heat in natural cavity flows".

J. of Fluid Mech. 37 (1969), p. 115-127.

8. MICHEL J.M. "Sillages plans supercavitants. Etude physique".

These. Grenoble, Mars 1973.

9. FOUULLT J. "Théorie asymptotiaue des écoulements supercavitants".

These. Paris, Novembre 1973.

10. MCHEL JM. - JODELET F. "Géométrie des cavités en écoulement supercavitant permanent ou périodi. que".

C.P.A.S. 273 p. $521-524$ (20 sept. 1971).

11. WU T.Y.T. "Unsteady supercavitating flows". 2nd Symo. on Nav. Hyd. (1958), p. 293-313.

12. BENJAMIN T.B. "Note on the interpretation of two dimensional theories of growing cavities". J. of Fiuid Mech. 19.1 (1964), p. 137-144.

13. MICHEL J.M. "Ventilated cavities. A contribution to the study of pulsation mechanism". Symp. I.U.T.A.M. Leningrad, Juin 1971. 